School Integration Limits the Ability of Local Norms to Diversify Gifted Programs: A Mathematical Analysis with Implications Related to the Achievement Gap

\author{
Russell T. Warne ${ }^{1}$ and Ross A. A. Larsen ${ }^{2}$ \\ ${ }^{1}$ Department of Behavioral Science, Utah Valley University \\ ${ }^{2}$ Department of Instructional and Technology, Brigham Young University
}

\begin{abstract}
Author Note
Russell T. Warne (D) https://orcid.org/0000-0003-4763-3625

Ross A. A. Larsen (D https://orcid.org/0000-0002-5924-3993

We have no conflicts of interest to disclose. Alicia McIntire contributed to the simulation code. We greatly appreciate the feedback from Dr. Dante Dixson on an early draft of this article. Correspondence concerning this article should be addressed to Russell T. Warne, $800 \mathrm{~W}$. University Parkway MC 115, Orem, UT 84058. Email: rwarne@uvu.edu
\end{abstract}




\begin{abstract}
Experts within gifted education have advocated for the use of local norms when selecting students for gifted programs, instead of national-level norms. Local norms compare students to their immediate peers to identify gifted students and are believed to produce a more diverse gifted program. However, district integration limits the ability of local norms to diversify gifted programs, a fact which has been almost completely overlooked in gifted education scholarship. Through a simplified example, we show that local building-level norms are best at diversifying gifted programs when schools are highly segregated. Conversely, when achievement gaps are present and a uniform admissions cutoff is applied, building-level norms in highly integrated schools produce highly segregated gifted programs. In short, the use of building-level local norms trades one form of segregation for another. Implications and recommendations for gifted education and beyond are explored. A preprint version of this article is available at https://psyarxiv.com/nemch.
\end{abstract}

Keywords: gifted identification, local norms, diversity, gifted education 


\section{School Integration Limits the Ability of Local Norms to Diversify Gifted Programs: A Mathematical Analysis with Implications Related to the Achievement Gap}

In the efforts to diversify gifted programs, one proposal that has emerged in the 2010s has been the suggested use of local norms - instead of national norms or the norms produced by a testing company—when selecting children for admission to gifted programs. This proposal has become mainstream in gifted education, and is now seen as best practice (e.g., Peters et al., 2014; Worrell \& Dixson, 2018).

Local norms have advantages over national or test norms when selecting children for gifted programs. First, local norms reflect the fact that education is a local endeavor and that gifted services are needed when a student's performance far exceeds that of his or her peers (Peters et al., 2014). A child in a high performing district may obtain test scores in the top 5\% nationwide in academic achievement but at the 70th percentile in the local district and have their needs met by lessons that are aimed at the middle range of achievement within their classroom. Conversely, a student in a school or district with a lower level of average achievement may be elite compared to her or his classmates, even though — compared to nationwide test norms — the child's test performance is at the 75 th percentile. In short, a decision to use national norms to select children for gifted programs is a decision to ignore the local context and the degree to which a child is outperforming the peers they see every day in school.

Second, local norms have the virtue of comparing children to those with a similar background as themselves. Undoubtedly, a child's environment—particularly educational opportunities - impact her or his performance on the measures that are used to select a child for a gifted program. These environmental characteristics that foster intellectual and academic 
development are not distributed evenly throughout society. Local norms-especially those that compare a child only to others who attend the same school or same classroom — greatly increase the likelihood that students are being compared to children with similar educational opportunities (Peters et al., 2019; Peters \& Engerrand, 2016). Thus, local norms are less influenced by relative environmental advantage and much more likely to measure a child's success in using the educational opportunities that are afforded to them.

Third, local norms can make it easier to allocate resources to serve gifted children. Yearto-year numbers of identified students are stable if children are selected for gifted programs using building-level norms because all buildings within a school district contribute the same number of students annually. This allows administrators to allocate staff and make plans for educational programs much more easily (Peters et al., 2019). Moreover, if local norms identify the same percentage of children in different buildings as gifted, then resources can also be distributed more equitably, thereby ensuring that gifted education does not exasperate interschool inequalities. For example, an enrichment program can be established at each building, and this program would serve the same percentage of children, allowing gifted education staff and budget to be split evenly across all buildings. If children are selected for a magnet program with building-level norms, then such a program would not reinforce pre-existing differences in access to advanced educational practices. However, it is important to recognize that some fixed overhead costs exist in creating and maintaining a gifted program (e.g., transportation, testing), and local norms will not alleviate the distribution of these expenditures.

Finally, some gifted education theorists have suggested that local norms could increase diversity of gifted programs and increase the likelihood that underrepresented studentsparticularly Hispanic and African American students - are selected for gifted programs. There is 
empirical support for this claim. In a study of academic achievement test data from over 3 million children in over 3,000 school districts (the largest study of its kind), Peters et al. (2019) found that using building-level local norms to label the top 5\% or top $15 \%$ of students as gifted improved diversity of the gifted population compared to using a uniform cutoff score across all buildings that resulted in the same overall percentage of children being labeled as gifted. Carman et al. $(2018,2020)$ used aptitude test scores on the Cognitive Abilities Test and the Naglieri Nonverbal Ability Test from over 15,000 children per cohort in an urban school district to examine the impact of using different norms to identify gifted children. They found that Hispanic and African American students would qualify for gifted programs at the highest rates when using school-level local norms — and at the lowest rates when using national norms.

However, the results of these studies (Carman et al., 2018, 2020; Peters et al., 2019) showed that, while diversity did improve, local building-level norms would not result in a gifted group that was representative of the larger district population. The ability for local norms to diversify gifted programs was limited, with Peters et al. (2019) concluding that "building norms will not increase the diversity within every building's gifted population. Rather, building norms have the greatest effect ... in schools with larger-than-average populations of minority students" (p. 15).

The purpose of this paper is to expound upon this finding and describe the limits of the ability of local norms to diversify a gifted program. The simulation in this article shows that Carman et al.'s $(2018,2020)$ and Peters et al.'s (2019) results can be explained by local norms' ability to diversify gifted programs is limited by (1) the degree of racial integration in school buildings and (2) average differences in scores. Carman et al. (2020) briefly mentioned that 
school integration can have an impact on the effectiveness of local school-level norms to create a gifted program that is representative of a school district's general population, stating,

The primary reason that school-level norms work so well at reducing the differences in identification rates between groups in highly diverse districts is most likely due to institutional- and district-imposed segregation. ... School-level norming would not work as efficiently for schools that were not already segregated for whatever reason. (Carman et al., 2020, pp. 189, 190)

This article bolsters Carman et al.'s (2020) claim that a district's level of racial integration places a direct limit on the effectiveness of local building-level norms to create racial integrated gifted programs. In this article, we will also show that the limits of local-norms to diversify gifted programs are a direct consequence of applying a uniform cutoff to a population that has achievement gaps among groups. We will also explore this phenomenon's implications for scholars and practitioners.

\section{Statistical Methods}

\section{Simulation Assumptions}

The simulation in this article are based on a few reasonable assumptions. The first is that selection for a gifted program can be collapsed to a single normally distributed variable. This is not to say that students are selected for a gifted program on the basis of a single score (e.g., an IQ score), but rather that whatever criteria are used to select children measure a single underlying giftedness construct that is unidimensional in nature, though manifested in different scores. This is, theoretically what occurs when selecting students for a gifted program by averaging scores across different measures (e.g., McBee et al., 2014) or using an identification matrix. This view of an underlying construct manifested with different scores is also the theory behind using an 
overall score on an aptitude or achievement test battery that has positively correlated subscores (Warne, 2016a, 2020).

The second assumption in the simulation is that the score distributions of different racial groups have the same standard deviation and only differ in their averages. While slight differences in standard deviations may cause disproportionate representation in groups of people at the extremes of a distribution (Deary et al., 2003; Feingold, 1992; Warne, 2020), no one within gifted education has proposed this as a major cause of disproportionality of selection of different racial groups into gifted programs. Instead, the mean differences across groups have a much larger impact on the representativeness of gifted programs, and controlling for the achievement gap sometimes statistically explains most or all of the disproportionality found in gifted programs (e.g., Warne et al., 2013). This has led some scholars to suggest that the disproportionality in gifted programs is a consequence of the overall achievement gap in academic achievement across racial groups (e.g., Worrell et al., 2019; Worrell \& Dixson, 2018).

The final assumption in this simulation is that — within a school — the same cutoff score is applied to students, regardless of their ethnic or racial group membership. In other words, the selection process within a school would be colorblind, and all students who exceed the school's cutoff are selected for the gifted program. Peters et al. (2019) and Carman et al. (2018, 2020) used a similar uniform standard when applying local norms to identifying gifted children.

\section{Simulation Parameters}

To show how school racial integration limits the ability for local norms to diversify gifted programs, we conducted a simulation study that varies three characteristics of a school district: the size of average group differences, the level of segregation in district schools, and the cutoff score for admission to a gifted program. 
Average group differences. Average score differences (e.g., mean achievement gaps) among demographic groups vary greatly and depend on the groups being compared, the test(s) used to measure differences, and local conditions. In the most comprehensive examination of score gaps, Reardon et al. (2019) found that the average achievement gap between White and African American students in American school districts was $d=0.66$, but that this mean difference ranged from a minimum of $d \approx .20$ in the most equitable districts to $d \approx 1.25$ in the districts that had the greatest achievement gaps. Gaps between White and Hispanic students averaged $d=.50$, but the range of gaps was similarly wide: $d \approx .10$ to 1.15 .

We simulated achievement gaps ranging from $d=0$ to $d=2.0$ in increments of 0.10 standard deviations, resulting in 21 conditions for this variable. For convenience, the racial group with the higher average will be called Group A, and the racial group with the lower mean academic achievement will be Group B.

Selected percentage. In this simulation, we examined the impact of selecting different percentages of students for gifted programs. There were six percentages of students: the top 20\%, top $15 \%$, top $10 \%$, top $5 \%$, top $2 \%$, and top $1 \%$ of all students within a school. Like several other investigations of gifted identification (e.g., Carman et al., 2018, 2020; Lakin, 2018; Peters et al., 2019), all students whose scores are equal to or above this cutoff will be labeled as gifted, and all students below the cutoff within a building will be the non-gifted group.

District racial integration. For these calculations, there are 11 levels of racial integration, ranging from schools that are fully racially integrated to being completely segregated. The degree of integration is measured with the index of dissimilarity, which is calculated by finding the difference for each school between its proportion of students in a minority racial group from the overall proportion in the entire district. These differences are then 
weighted by overall school size and summed (James \& Taueber, 1985, Formula 2). The result is a number ranging from 0 to 1 that indicates the proportion of students from a demographic group that would have to change schools for all schools to be fully integrated and have a demographic makeup that perfectly reflects the overall district demographics. This metric has been used in other studies of school segregation (e.g., Kalogrides \& Loeb, 2013) and has an intuitive interpretation (James \& Taueber, 1985). A dissimilarity index of 1.0 indicates complete segregation, while a dissimilarity index of zero indicates that all schools within a district are perfectly integrated and have a student body that exactly reflects the district's overall student population. 
Table 1

Demographic Composition of Districts in the Calculations

\begin{tabular}{|c|c|c|c|c|c|c|c|c|c|c|c|}
\hline & District 1 & District 2 & District 3 & District 4 & District 5 & District 6 & District 7 & District 8 & District 9 & District 10 & District 11 \\
\hline School 1 & $100 \% / 0 \%$ & $100 \% / 0 \%$ & $90 \% / 10 \%$ & $90 \% / 10 \%$ & $80 \% / 20 \%$ & $80 \% / 20 \%$ & $70 \% / 30 \%$ & $70 \% / 30 \%$ & $60 \% / 40 \%$ & $60 \% / 40 \%$ & $50 \% / 50 \%$ \\
\hline School 2 & $100 \% / 0 \%$ & $90 \% / 10 \%$ & $90 \% / 10 \%$ & $80 \% / 20 \%$ & $80 \% / 20 \%$ & $70 \% / 30 \%$ & $70 \% / 30 \%$ & $60 \% / 40 \%$ & $60 \% / 40 \%$ & $50 \% / 50 \%$ & $50 \% / 50 \%$ \\
\hline School 3 & $0 \% / 100 \%$ & $10 \% / 90 \%$ & $10 \% / 90 \%$ & $20 \% / 80 \%$ & $20 \% / 80 \%$ & $30 \% / 70 \%$ & $30 \% / 70 \%$ & $40 \% / 60 \%$ & $40 \% / 60 \%$ & $50 \% / 50 \%$ & $50 \% / 50 \%$ \\
\hline School 4 & $0 \% / 100 \%$ & $0 \% / 100 \%$ & $10 \% / 90 \%$ & $10 \% / 90 \%$ & $20 \% / 80 \%$ & $80 \% / 20 \%$ & $30 \% / 70 \%$ & $30 \% / 70 \%$ & $40 \% / 60 \%$ & $40 \% / 60 \%$ & $50 \% / 50 \%$ \\
\hline $\begin{array}{l}\text { Dissimilarity } \\
\text { Index }\end{array}$ & 1.00 & 0.90 & 0.80 & 0.70 & 0.60 & 0.50 & 0.40 & 0.30 & 0.20 & 0.10 & 0.00 \\
\hline
\end{tabular}

Note. Composition is listed as Group A/Group B.

Note. All schools are the same size, and the overall district composition for all districts is $50 \% / 50 \%$. 
In this study, we simulated 11 levels of district-level segregation, with dissimilarity indexes ranging from 0 to 1.0 in increments of 0.1 . In all simulated districts, there were four school buildings with the same number of students enrolled at each campus. Table 1 shows the demographic composition for the hypothetical school districts in the simulation. All districts have $50 \%$ of their student body belonging to Group A and 50\% in Group B, and all districts consist of four schools with the same number of enrolled students. District 1 is a completely segregated district, with all the students in the higher scoring group enrolled in two schools and all the students in the lower scoring group enrolled in the other two schools, leading to a dissimilarity index of 1.00. Districts become increasingly desegregated until reaching District 11, which is perfectly integrated and has a dissimilarity index of zero. For reference, a dissimilarity index of 0.70 or 0.80 is found in the most segregated school districts in the United States (Frisby, 2013, Tables 4.3-4.5). Dissimilarity indexes tend to be higher in urban districts and lowest in rural districts. Fry (2009, Table 7) reported that in the 2006-2007 school year, the average dissimilarity index for African American students was 0.44 in urban districts, 0.35 in suburban districts, and 0.27 in rural districts. For Hispanic students the mean dissimilarity indexes were 0.38 in urban districts, 0.30 in suburban districts, and 0.21 in rural districts. Finally, for Asian students, the average dissimilarity index as 0.33 in urban districts, 0.25 in suburban districts, and 0.24 in rural districts.

\section{Calculation Procedures and Dependent Variables}

Given the conditions listed for the three independent variables, this simulation has 1,386 unique combinations of conditions $(21 \times 6 \times 11=1,386)$ that was run 500 times per combination of conditions. The simulation procedure for each combination of conditions was as follows. First, 
within each building two normal distributions with the same standard deviation, consisting of 500 students' scores were created, one for Group A and another for Group B, with the latter distribution having a mean that was lower than Group A's mean, as determined by the mean score difference for that condition. (Note that this means that every school in the simulation has a total enrollment of 1,000 students.) Second, a cutoff was set so that the top $x \%$ of scores within each school were labeled as being selected for a gifted program. The selected groups from each school were combined to examine the overall makeup of the gifted population. The $\mathrm{R}$ code for generating the data and selecting the simulated gifted children is included in the article's supplemental files.

Using these scores, the following statistics were examined as dependent variables:

1. The demographic makeup of the group selected for a gifted program. This is the percentage of the gifted group that belongs to Group A and the percentage of the gifted group that belongs to Group B.

2. The average $z$-score of the gifted group. This was calculated by finding the average $z-$ score of all sample members who were labeled as gifted. For this z-score, zero corresponds to the average score for all students in the district who belong to Group A.

3. The mean score difference between gifted Group A individuals and gifted Group B individuals. This is reported as a Cohen's $d$ value that expresses the number of standard deviations between the two groups' means, calculated with data only from individuals labeled as gifted. The unit for this statistic is the standard deviation for all Group A students in a district (which is equal to the standard deviation for all Group B students in a district). 
4. The relative risks for students from Group A and Group B being selected for the gifted program. For Group A, this is the proportion of group members labeled as gifted divided by the proportion of Group B members who are labeled as gifted. For Group B, this is the proportion of group members who are identified as gifted divided by the proportion of Group A members who are labeled as gifted. A value of 1.0 indicates that members of both groups are equally likely to be labeled as gifted. Values higher than 1.0 mean that members of a group are more likely than the other group to be identified as gifted; values lower than 1.0 indicate that members of a group are less likely to be labeled as gifted. Note that these two relative risks are reciprocals or each other (see Warne, 2021, Chapter 14). Although one is mathematically redundant, both are presented here for the reader's convenience.

5. The gifted/non-gifted dissimilarity index within the district. This statistic is a measure of the racial segregation comparing the demographics of the gifted program with the demographics of the students not selected for the gifted program and treats these two groups as if they were two separate schools within the district. Just as with the district dissimilarity indexes, a dissimilarity index of zero indicates that a gifted program is perfectly integrated (and therefore reflects the demographics of the district). Higher values indicate greater degrees of segregation between gifted and non-gifted groups.

\section{Results}

\section{Calculation Results}

Tables 2-6 display selected results of the simulation, showing for each district the demographics of the gifted group, average $z$-score of the group selected for the gifted program, the mean difference between demographic groups among children in the gifted program, the 
relative risk for each group to be selected for the program, and a gifted/non-gifted dissimilarity index that quantifies the level of segregation when comparing the gifted students with all nongifted students. Table 2 displays these statistics when $d=0$, i.e., there is no mean achievement gap. Tables 3-6 display the same information gap for $d=.30, .60, .90$, and 1.20, respectively. Similar tables for other achievement gap sizes (ranging from $d=0$ to 2.0 in increments of .1) are available from the supplementary files for this article. 
Table 2

Estimated Gifted Program Data, Under Varying Percentages Selected for Gifted Programs and Levels of District Segregation When $d=0$

\begin{tabular}{|c|c|c|c|c|c|c|c|c|c|c|c|}
\hline & District 1 & District 2 & District 3 & District 4 & District 5 & District 6 & District 7 & District 8 & District 9 & District 10 & District 11 \\
\hline \multicolumn{12}{|l|}{ Top 20\% } \\
\hline Gifted $\%$ in Group A & $50.0 \%$ & $50.0 \%$ & $50.0 \%$ & $50.0 \%$ & $50.0 \%$ & $50.0 \%$ & $49.9 \%$ & $50.0 \%$ & $50.0 \%$ & $50.0 \%$ & $49.9 \%$ \\
\hline Gifted $\%$ in Group B & $50.0 \%$ & $50.0 \%$ & $50.0 \%$ & $50.0 \%$ & $50.0 \%$ & $50.0 \%$ & $50.1 \%$ & $50.0 \%$ & $50.0 \%$ & $50.0 \%$ & $50.0 \%$ \\
\hline Average $z$-score for Gifted Group & 1.40 & 1.40 & 1.40 & 1.40 & 1.40 & 1.40 & 1.40 & 1.40 & 1.40 & 1.40 & 1.40 \\
\hline $\begin{array}{l}\text { Gifted Group Mean Difference } \\
\text { Relative Risk of Identification for }\end{array}$ & 0.00 & 0.00 & 0.00 & 0.00 & 0.00 & 0.00 & 0.00 & 0.00 & 0.00 & 0.00 & 0.00 \\
\hline $\begin{array}{l}\text { Group A } \\
\text { Relative Risk of Identification for }\end{array}$ & 1.00 & 1.00 & 1.00 & 1.00 & 1.00 & 1.00 & 1.00 & 1.00 & 1.00 & 1.00 & 0.99 \\
\hline $\begin{array}{l}\text { Group B } \\
\text { Dissimilarity Index (Gifted/Non- }\end{array}$ & 1.00 & 1.00 & 1.00 & 1.00 & 1.00 & 1.00 & 1.00 & 1.00 & 1.00 & 1.00 & 1.01 \\
\hline Gifted) & 0.00 & 0.00 & 0.00 & 0.00 & 0.00 & 0.00 & 0.00 & 0.00 & 0.00 & 0.00 & 0.00 \\
\hline \multicolumn{12}{|l|}{ Тор $15 \%$} \\
\hline Gifted \% in Group A & $50.0 \%$ & $50.0 \%$ & $50.1 \%$ & $50.0 \%$ & $49.9 \%$ & $50.0 \%$ & $49.9 \%$ & $50.0 \%$ & $50.1 \%$ & $49.9 \%$ & $49.9 \%$ \\
\hline Gifted \% in Group B & $50.0 \%$ & $50.0 \%$ & $49.9 \%$ & $50.0 \%$ & $50.1 \%$ & $50.0 \%$ & $50.1 \%$ & $50.0 \%$ & $49.9 \%$ & $50.1 \%$ & $50.1 \%$ \\
\hline Average $z$-score for Gifted Group & 1.55 & 1.55 & 1.56 & 1.55 & 1.55 & 1.55 & 1.55 & 1.55 & 1.55 & 1.56 & 1.55 \\
\hline $\begin{array}{l}\text { Gifted Group Mean Difference } \\
\text { Relative Risk of Identification for }\end{array}$ & 0.00 & 0.00 & 0.00 & 0.00 & 0.00 & 0.00 & 0.00 & 0.00 & 0.00 & 0.00 & 0.00 \\
\hline $\begin{array}{l}\text { Group A } \\
\text { Relative Risk of Identification for }\end{array}$ & 1.00 & 1.00 & 1.00 & 1.00 & 1.00 & 1.00 & 1.00 & 1.00 & 1.00 & 1.00 & 0.99 \\
\hline $\begin{array}{l}\text { Group B } \\
\text { Dissimilarity Index (Gifted/Non- }\end{array}$ & 1.00 & 1.00 & 1.00 & 1.00 & 1.00 & 1.00 & 1.00 & 1.00 & 1.00 & 1.00 & 1.01 \\
\hline Gifted) & 0.00 & 0.00 & 0.00 & 0.00 & 0.00 & 0.00 & 0.00 & 0.00 & 0.00 & 0.00 & 0.00 \\
\hline \multicolumn{12}{|l|}{ Тор $10 \%$} \\
\hline Gifted $\%$ in Group A & $50.0 \%$ & $50.0 \%$ & $50.2 \%$ & $50.0 \%$ & $50.0 \%$ & $50.0 \%$ & $49.9 \%$ & $50.0 \%$ & $50.1 \%$ & $49.9 \%$ & $50.0 \%$ \\
\hline Gifted $\%$ in Group B & $50.0 \%$ & $50.0 \%$ & $49.8 \%$ & $50.0 \%$ & $50.0 \%$ & $50.0 \%$ & $50.1 \%$ & $50.0 \%$ & $49.9 \%$ & $50.1 \%$ & $50.0 \%$ \\
\hline Average $z$-score for Gifted Group & 1.75 & 1.75 & 1.76 & 1.75 & 1.75 & 1.75 & 1.75 & 1.75 & 1.76 & 1.76 & 1.75 \\
\hline $\begin{array}{l}\text { Gifted Group Mean Difference } \\
\text { Relative Risk of Identification for }\end{array}$ & 0.00 & 0.01 & 0.00 & 0.00 & 0.00 & 0.00 & 0.00 & 0.00 & 0.00 & 0.00 & 0.00 \\
\hline $\begin{array}{l}\text { Group A } \\
\text { Relative Risk of Identification for }\end{array}$ & 1.00 & 1.00 & 1.01 & 1.00 & 1.00 & 1.00 & 1.00 & 1.00 & 1.00 & 1.00 & 1.00 \\
\hline $\begin{array}{l}\text { Group B } \\
\text { Dissimilarity Index (Gifted/Non- }\end{array}$ & 1.00 & 1.00 & 0.99 & 1.00 & 1.00 & 1.00 & 1.00 & 1.00 & 1.00 & 1.00 & 1.00 \\
\hline Gifted) & 0.00 & 0.00 & 0.00 & 0.00 & 0.00 & 0.00 & 0.00 & 0.00 & 0.00 & 0.00 & 0.00 \\
\hline \multicolumn{12}{|l|}{ Top 5\% } \\
\hline Gifted $\%$ in Group A & $50.0 \%$ & $50.0 \%$ & $50.2 \%$ & $50.1 \%$ & $49.9 \%$ & $49.9 \%$ & $49.9 \%$ & $50.0 \%$ & $50.3 \%$ & $50.1 \%$ & $50.0 \%$ \\
\hline Gifted $\%$ in Group B & $50.0 \%$ & $50.0 \%$ & $49.8 \%$ & $49.9 \%$ & $50.1 \%$ & $50.1 \%$ & $50.1 \%$ & $50.0 \%$ & $49.7 \%$ & $49.9 \%$ & $50.0 \%$ \\
\hline Average $z$-score for Gifted Group & 2.06 & 2.06 & 2.06 & 2.06 & 2.06 & 2.06 & 2.06 & 2.06 & 2.06 & 2.07 & 2.06 \\
\hline Gifted Group Mean Difference & 0.00 & 0.00 & 0.00 & 0.00 & -0.01 & 0.00 & 0.00 & 0.00 & 0.00 & 0.00 & 0.00 \\
\hline
\end{tabular}


Relative Risk of Identification for Group A

Relative Risk of Identification for Group B

Dissimilarity Index (Gifted/NonGifted)

\begin{tabular}{|c|c|c|c|c|c|c|c|c|c|c|}
\hline 1.00 & 1.00 & 1.01 & 1.00 & 1.00 & 1.00 & 0.99 & 1.00 & 1.01 & 1.00 & 1.00 \\
\hline 1.00 & 1.00 & 0.99 & 1.00 & 1.00 & 1.00 & 1.01 & 1.00 & 0.99 & 1.00 & 1.00 \\
\hline 0.00 & 0.00 & 0.00 & 0.00 & 0.00 & 0.00 & 0.00 & 0.00 & 0.00 & 0.00 & 0.00 \\
\hline $50.0 \%$ & $50.0 \%$ & $50.3 \%$ & $50.1 \%$ & $49.8 \%$ & $49.8 \%$ & $49.8 \%$ & $50.0 \%$ & $50.0 \%$ & $49.8 \%$ & $50.2 \%$ \\
\hline $50.0 \%$ & $50.0 \%$ & $49.7 \%$ & $49.9 \%$ & $50.2 \%$ & $50.2 \%$ & $50.2 \%$ & $50.0 \%$ & $50.0 \%$ & $50.2 \%$ & $49.8 \%$ \\
\hline 2.42 & 2.42 & 2.42 & 2.42 & 2.42 & 2.42 & 2.42 & 2.42 & 2.42 & 2.43 & 2.42 \\
\hline 0.00 & 0.00 & 0.00 & 0.00 & 0.00 & 0.00 & 0.00 & 0.01 & 0.00 & 0.00 & 0.00 \\
\hline 1.00 & 1.00 & 1.01 & 1.00 & 0.99 & 0.99 & 0.99 & 1.00 & 1.00 & 0.99 & 1.01 \\
\hline 1.00 & 1.00 & 0.99 & 1.00 & 1.01 & 1.01 & 1.01 & 1.00 & 1.00 & 1.01 & 0.99 \\
\hline 0.00 & 0.00 & 0.00 & 0.00 & 0.00 & 0.00 & 0.00 & 0.00 & 0.00 & 0.00 & 0.00 \\
\hline $50.0 \%$ & $50.0 \%$ & $50.3 \%$ & $49.8 \%$ & $49.7 \%$ & $49.7 \%$ & $49.9 \%$ & $50.5 \%$ & $50.2 \%$ & $50.0 \%$ & $50.1 \%$ \\
\hline $50.0 \%$ & $50.0 \%$ & $49.7 \%$ & $50.2 \%$ & $50.3 \%$ & $50.3 \%$ & $50.1 \%$ & $49.5 \%$ & $49.8 \%$ & $50.0 \%$ & $49.9 \%$ \\
\hline 2.66 & 2.66 & 2.67 & 2.67 & 2.67 & 2.67 & 2.67 & 2.66 & 2.67 & 2.67 & 2.67 \\
\hline 0.00 & 0.00 & 0.00 & 0.00 & 0.00 & 0.00 & 0.00 & 0.00 & 0.00 & 0.00 & 0.01 \\
\hline 1.00 & 1.00 & 1.01 & 0.99 & 0.99 & 0.99 & 0.99 & 1.02 & 1.01 & 1.00 & 1.00 \\
\hline 1.00 & 1.00 & 0.99 & 1.01 & 1.01 & 1.01 & 1.01 & 0.98 & 0.99 & 1.00 & 1.00 \\
\hline 0.00 & 0.00 & 0.00 & 0.00 & 0.00 & 0.00 & 0.00 & 0.00 & 0.00 & 0.00 & 0.00 \\
\hline
\end{tabular}

Top 2\%

Gifted \% in Group A

Gifted \% in Group B

Average $z$-score for Gifted Group

Gifted Group Mean Difference

Relative Risk of Identification for Group A

Relative Risk of Identification for Group B

Dissimilarity Index (Gifted/Non-

Gifted)

Top $1 \%$

Gifted \% in Group A

Gifted $\%$ in Group B

Average $z$-score for Gifted Group

Gifted Group Mean Difference

Relative Risk of Identification for Group A

Relative Risk of Identification for Group B

Dissimilarity Index (Gifted/Non-

Gifted) 
Table 3

Estimated Gifted Program Data, Under Varying Percentages Selected for Gifted Programs and Levels of District Segregation When $d=0.3$

\begin{tabular}{|c|c|c|c|c|c|c|c|c|c|c|c|}
\hline & District 1 & District 2 & District 3 & District 4 & District 5 & District 6 & District 7 & District 8 & District 9 & District 10 & District 11 \\
\hline \multicolumn{12}{|l|}{ Top $20 \%$} \\
\hline Gifted $\%$ in Group A & $50.0 \%$ & $51.9 \%$ & $53.7 \%$ & $55.1 \%$ & $56.6 \%$ & $57.7 \%$ & $58.7 \%$ & $59.2 \%$ & $60.0 \%$ & $60.3 \%$ & $60.3 \%$ \\
\hline Gifted $\%$ in Group B & $50.0 \%$ & $48.1 \%$ & $46.3 \%$ & $44.9 \%$ & $43.4 \%$ & $42.3 \%$ & $41.3 \%$ & $40.8 \%$ & $40.0 \%$ & $39.7 \%$ & $39.7 \%$ \\
\hline Average $z$-score for Gifted Group & 1.25 & 1.25 & 1.25 & 1.26 & 1.26 & 1.26 & 1.26 & 1.26 & 1.26 & 1.27 & 1.26 \\
\hline $\begin{array}{l}\text { Gifted Group Mean Difference } \\
\text { Relative Risk of Identification for }\end{array}$ & 0.30 & 0.26 & 0.22 & 0.19 & 0.15 & 0.13 & 0.10 & 0.09 & 0.07 & 0.07 & 0.07 \\
\hline $\begin{array}{l}\text { Group A } \\
\quad \text { Relative Risk of Identification for }\end{array}$ & 1.00 & 1.08 & 1.16 & 1.23 & 1.30 & 1.37 & 1.42 & 1.45 & 1.50 & 1.52 & 1.52 \\
\hline $\begin{array}{l}\text { Group B } \\
\text { Dissimilarity Index (Gifted/Non- }\end{array}$ & 1.00 & 0.93 & 0.86 & 0.81 & 0.77 & 0.73 & 0.70 & 0.69 & 0.67 & 0.66 & 0.66 \\
\hline Gifted) & 0.00 & 0.02 & 0.03 & 0.04 & 0.05 & 0.06 & 0.07 & 0.07 & 0.08 & 0.08 & 0.08 \\
\hline \multicolumn{12}{|l|}{ Top $15 \%$} \\
\hline Gifted $\%$ in Group A & $50.0 \%$ & $52.1 \%$ & $54.1 \%$ & $55.7 \%$ & $57.3 \%$ & $58.6 \%$ & $59.6 \%$ & $60.2 \%$ & $61.0 \%$ & $61.3 \%$ & $61.5 \%$ \\
\hline Gifted \% in Group B & $50.0 \%$ & $47.9 \%$ & $45.9 \%$ & $44.3 \%$ & $42.7 \%$ & $41.4 \%$ & $40.4 \%$ & $39.8 \%$ & $39.0 \%$ & $38.7 \%$ & $38.5 \%$ \\
\hline Average $z$-score for Gifted Group & 1.41 & 1.41 & 1.41 & 1.41 & 1.41 & 1.42 & 1.42 & 1.42 & 1.42 & 1.42 & 1.42 \\
\hline $\begin{array}{l}\text { Gifted Group Mean Difference } \\
\text { Relative Risk of Identification for }\end{array}$ & 0.30 & 0.25 & 0.21 & 0.18 & 0.14 & 0.13 & 0.10 & 0.08 & 0.07 & 0.06 & 0.06 \\
\hline $\begin{array}{l}\text { Group A } \\
\text { Relative Risk of Identification for }\end{array}$ & 1.00 & 1.09 & 1.18 & 1.26 & 1.34 & 1.42 & 1.48 & 1.51 & 1.57 & 1.59 & 1.60 \\
\hline $\begin{array}{l}\text { Group B } \\
\text { Dissimilarity Index (Gifted/Non- }\end{array}$ & 1.00 & 0.92 & 0.85 & 0.80 & 0.74 & 0.71 & 0.68 & 0.66 & 0.64 & 0.63 & 0.63 \\
\hline Gifted) & 0.00 & 0.01 & 0.02 & 0.03 & 0.04 & 0.05 & 0.06 & 0.06 & 0.07 & 0.07 & 0.07 \\
\hline \multicolumn{12}{|l|}{ Top $10 \%$} \\
\hline Gifted $\%$ in Group A & $50.0 \%$ & $52.4 \%$ & $54.7 \%$ & $56.5 \%$ & $58.2 \%$ & $59.7 \%$ & $60.9 \%$ & $61.5 \%$ & $62.3 \%$ & $62.7 \%$ & $63.0 \%$ \\
\hline Gifted \% in Group B & $50.0 \%$ & $47.6 \%$ & $45.3 \%$ & $43.5 \%$ & $41.8 \%$ & $40.3 \%$ & $39.1 \%$ & $38.5 \%$ & $37.7 \%$ & $37.3 \%$ & $37.0 \%$ \\
\hline Average $z$-score for Gifted Group & 1.61 & 1.61 & 1.61 & 1.61 & 1.62 & 1.62 & 1.62 & 1.62 & 1.62 & 1.63 & 1.62 \\
\hline $\begin{array}{l}\text { Gifted Group Mean Difference } \\
\text { Relative Risk of Identification for }\end{array}$ & 0.30 & 0.25 & 0.21 & 0.18 & 0.14 & 0.12 & 0.09 & 0.08 & 0.06 & 0.06 & 0.05 \\
\hline $\begin{array}{l}\text { Group A } \\
\text { Relative Risk of Identification for }\end{array}$ & 1.00 & 1.10 & 1.20 & 1.30 & 1.39 & 1.48 & 1.56 & 1.60 & 1.65 & 1.68 & 1.70 \\
\hline $\begin{array}{l}\text { Group B } \\
\text { Dissimilarity Index (Gifted/Non- }\end{array}$ & 1.00 & 0.91 & 0.83 & 0.77 & 0.72 & 0.68 & 0.64 & 0.62 & 0.61 & 0.60 & 0.59 \\
\hline Gifted) & 0.00 & 0.01 & 0.02 & 0.03 & 0.03 & 0.04 & 0.04 & 0.05 & 0.05 & 0.05 & 0.05 \\
\hline \multicolumn{12}{|l|}{ Top 5\% } \\
\hline Gifted $\%$ in Group A & $50.0 \%$ & $53.0 \%$ & $55.5 \%$ & $57.7 \%$ & $59.7 \%$ & $61.3 \%$ & $62.7 \%$ & $63.5 \%$ & $64.5 \%$ & $64.7 \%$ & $64.9 \%$ \\
\hline Gifted \% in Group B & $50.0 \%$ & $47.0 \%$ & $44.5 \%$ & $42.3 \%$ & $40.3 \%$ & $38.7 \%$ & $37.3 \%$ & $36.5 \%$ & $35.5 \%$ & $35.3 \%$ & $35.1 \%$ \\
\hline Average $z$-score for Gifted Group & 1.91 & 1.92 & 1.92 & 1.92 & 1.93 & 1.93 & 1.93 & 1.93 & 1.94 & 1.94 & 1.94 \\
\hline Gifted Group Mean Difference & 0.30 & 0.25 & 0.20 & 0.18 & 0.13 & 0.12 & 0.08 & 0.07 & 0.05 & 0.05 & 0.04 \\
\hline
\end{tabular}


Relative Risk of Identification for Group A

Relative Risk of Identification for Group B

Dissimilarity Index (Gifted/NonGifted)

\begin{tabular}{|c|c|c|c|c|c|c|c|c|c|c|}
\hline 1.00 & 1.13 & 1.25 & 1.36 & 1.48 & 1.58 & 1.68 & 1.74 & 1.82 & 1.84 & 1.85 \\
\hline 1.00 & 0.89 & 0.80 & 0.73 & 0.67 & 0.63 & 0.60 & 0.57 & 0.55 & 0.54 & 0.54 \\
\hline 0.00 & 0.01 & 0.01 & 0.02 & 0.02 & 0.02 & 0.03 & 0.03 & 0.03 & 0.03 & 0.03 \\
\hline $50.0 \%$ & $53.6 \%$ & $56.4 \%$ & $59.1 \%$ & $61.3 \%$ & $63.6 \%$ & $64.8 \%$ & $65.9 \%$ & $67.0 \%$ & $67.0 \%$ & $67.6 \%$ \\
\hline $50.0 \%$ & $46.4 \%$ & $43.6 \%$ & $40.9 \%$ & $38.7 \%$ & $36.4 \%$ & $35.2 \%$ & $34.1 \%$ & $33.0 \%$ & $33.0 \%$ & $32.4 \%$ \\
\hline 2.27 & 2.27 & 2.28 & 2.29 & 2.29 & 2.29 & 2.30 & 2.30 & 2.30 & 2.30 & 2.30 \\
\hline 0.30 & 0.24 & 0.20 & 0.17 & 0.13 & 0.11 & 0.07 & 0.06 & 0.04 & 0.04 & 0.03 \\
\hline 1.00 & 1.15 & 1.30 & 1.45 & 1.58 & 1.75 & 1.84 & 1.94 & 2.03 & 2.03 & 2.09 \\
\hline 1.00 & 0.87 & 0.77 & 0.69 & 0.63 & 0.57 & 0.54 & 0.52 & 0.49 & 0.49 & 0.48 \\
\hline 0.00 & 0.00 & 0.01 & 0.01 & 0.01 & 0.01 & 0.01 & 0.01 & 0.01 & 0.01 & 0.01 \\
\hline $50.0 \%$ & $53.9 \%$ & $57.1 \%$ & $60.1 \%$ & $62.3 \%$ & $65.1 \%$ & $66.1 \%$ & $67.9 \%$ & $68.7 \%$ & $68.4 \%$ & $69.1 \%$ \\
\hline $50.0 \%$ & $46.1 \%$ & $42.9 \%$ & $39.9 \%$ & $37.7 \%$ & $34.9 \%$ & $33.9 \%$ & $32.1 \%$ & $31.3 \%$ & $31.6 \%$ & $30.9 \%$ \\
\hline 2.51 & 2.52 & 2.52 & 2.53 & 2.53 & 2.54 & 2.54 & 2.54 & 2.54 & 2.55 & 2.55 \\
\hline 0.31 & 0.24 & 0.19 & 0.17 & 0.13 & 0.10 & 0.07 & 0.05 & 0.04 & 0.04 & 0.03 \\
\hline 1.00 & 1.17 & 1.33 & 1.51 & 1.65 & 1.87 & 1.95 & 2.11 & 2.19 & 2.17 & 2.24 \\
\hline 1.00 & 0.85 & 0.75 & 0.66 & 0.61 & 0.54 & 0.51 & 0.47 & 0.46 & 0.46 & 0.45 \\
\hline 0.00 & 0.00 & 0.00 & 0.00 & 0.00 & 0.01 & 0.01 & 0.01 & 0.01 & 0.01 & 0.01 \\
\hline
\end{tabular}

Top 2\%

Gifted \% in Group A

Gifted \% in Group B

Average $z$-score for Gifted Group

Gifted Group Mean Difference

Relative Risk of Identification for Group A

Relative Risk of Identification for Group B

Dissimilarity Index (Gifted/Non-

Gifted)

Top $1 \%$

Gifted \% in Group A

Gifted \% in Group B

Average $z$-score for Gifted Group

Gifted Group Mean Difference

Relative Risk of Identification for

Group A

Relative Risk of Identification for Group B

Dissimilarity Index (Gifted/Non-

Gifted) 
Table 4

Estimated Gifted Program Data, Under Varying Percentages Selected for Gifted Programs and Levels of District Segregation When d=0.6

\begin{tabular}{|c|c|c|c|c|c|c|c|c|c|c|c|}
\hline & District 1 & District 2 & District 3 & District 4 & District 5 & District 6 & District 7 & District 8 & District 9 & District 10 & District 11 \\
\hline \multicolumn{12}{|l|}{ Top 20\% } \\
\hline Gifted \% in Group A & $50.0 \%$ & $53.7 \%$ & $57.4 \%$ & $60.3 \%$ & $63.0 \%$ & $65.1 \%$ & $67.1 \%$ & $68.3 \%$ & $69.5 \%$ & $69.9 \%$ & $70.3 \%$ \\
\hline Gifted \% in Group B & $50.0 \%$ & $46.3 \%$ & $42.6 \%$ & $39.7 \%$ & $37.0 \%$ & $34.9 \%$ & $32.9 \%$ & $31.7 \%$ & $30.5 \%$ & $30.1 \%$ & $29.7 \%$ \\
\hline Average $z$-score for Gifted Group & 1.10 & 1.11 & 1.12 & 1.13 & 1.14 & 1.15 & 1.15 & 1.15 & 1.16 & 1.16 & 1.16 \\
\hline $\begin{array}{l}\text { Gifted Group Mean Difference } \\
\text { Relative Risk of Identification for }\end{array}$ & 0.60 & 0.52 & 0.43 & 0.36 & 0.30 & 0.26 & 0.21 & 0.18 & 0.15 & 0.14 & 0.13 \\
\hline $\begin{array}{l}\text { Group A } \\
\text { Relative Risk of Identification for }\end{array}$ & 1.00 & 1.16 & 1.35 & 1.52 & 1.70 & 1.86 & 2.04 & 2.15 & 2.27 & 2.32 & 2.37 \\
\hline $\begin{array}{l}\text { Group B } \\
\text { Dissimilarity Index (Gifted/Non- }\end{array}$ & 1.00 & 0.86 & 0.74 & 0.66 & 0.59 & 0.54 & 0.49 & 0.46 & 0.44 & 0.43 & 0.42 \\
\hline Gifted) & 0.00 & 0.03 & 0.06 & 0.08 & 0.10 & 0.12 & 0.14 & 0.15 & 0.16 & 0.16 & 0.16 \\
\hline \multicolumn{12}{|l|}{ Тор $15 \%$} \\
\hline Gifted \% in Group A & $50.0 \%$ & $54.1 \%$ & $58.3 \%$ & $61.4 \%$ & $64.6 \%$ & $66.7 \%$ & $68.9 \%$ & $70.2 \%$ & $71.5 \%$ & $71.9 \%$ & $72.3 \%$ \\
\hline Gifted \% in Group B & $50.0 \%$ & $45.9 \%$ & $41.7 \%$ & $38.6 \%$ & $35.4 \%$ & $33.3 \%$ & $31.1 \%$ & $29.8 \%$ & $28.5 \%$ & $28.1 \%$ & $27.7 \%$ \\
\hline Average $z$-score for Gifted Group & 1.25 & 1.27 & 1.28 & 1.29 & 1.30 & 1.31 & 1.31 & 1.31 & 1.32 & 1.32 & 1.32 \\
\hline $\begin{array}{l}\text { Gifted Group Mean Difference } \\
\text { Relative Risk of Identification for }\end{array}$ & 0.60 & 0.52 & 0.42 & 0.35 & 0.29 & 0.25 & 0.20 & 0.17 & 0.13 & 0.12 & 0.11 \\
\hline $\begin{array}{l}\text { Group A } \\
\quad \text { Relative Risk of Identification for }\end{array}$ & 1.00 & 1.18 & 1.40 & 1.59 & 1.82 & 2.00 & 2.22 & 2.36 & 2.51 & 2.55 & 2.61 \\
\hline $\begin{array}{l}\text { Group B } \\
\text { Dissimilarity Index (Gifted/Non- }\end{array}$ & 1.00 & 0.85 & 0.72 & 0.63 & 0.55 & 0.50 & 0.45 & 0.42 & 0.40 & 0.39 & 0.38 \\
\hline Gifted) & 0.00 & 0.02 & 0.05 & 0.07 & 0.09 & 0.10 & 0.11 & 0.12 & 0.13 & 0.13 & 0.13 \\
\hline \multicolumn{12}{|l|}{ Тор $10 \%$} \\
\hline Gifted $\%$ in Group A & $50.0 \%$ & $54.8 \%$ & $59.5 \%$ & $63.0 \%$ & $66.5 \%$ & $68.9 \%$ & $71.3 \%$ & $72.6 \%$ & $73.9 \%$ & $74.3 \%$ & $74.8 \%$ \\
\hline Gifted $\%$ in Group B & $50.0 \%$ & $45.2 \%$ & $40.5 \%$ & $37.0 \%$ & $33.5 \%$ & $31.1 \%$ & $28.7 \%$ & $27.4 \%$ & $26.1 \%$ & $25.7 \%$ & $25.2 \%$ \\
\hline Average $z$-score for Gifted Group & 1.45 & 1.47 & 1.48 & 1.49 & 1.50 & 1.51 & 1.52 & 1.52 & 1.53 & 1.53 & 1.53 \\
\hline $\begin{array}{l}\text { Gifted Group Mean Difference } \\
\text { Relative Risk of Identification for }\end{array}$ & 0.60 & 0.51 & 0.41 & 0.34 & 0.27 & 0.23 & 0.18 & 0.15 & 0.12 & 0.11 & 0.10 \\
\hline $\begin{array}{l}\text { Group A } \\
\quad \text { Relative Risk of Identification for }\end{array}$ & 1.00 & 1.21 & 1.47 & 1.70 & 1.99 & 2.21 & 2.48 & 2.65 & 2.83 & 2.89 & 2.96 \\
\hline $\begin{array}{l}\text { Group B } \\
\text { Dissimilarity Index (Gifted/Non- }\end{array}$ & 1.00 & 0.82 & 0.68 & 0.59 & 0.50 & 0.45 & 0.40 & 0.38 & 0.35 & 0.35 & 0.34 \\
\hline Gifted) & 0.00 & 0.02 & 0.04 & 0.05 & 0.07 & 0.08 & 0.09 & 0.09 & 0.10 & 0.10 & 0.10 \\
\hline \multicolumn{12}{|l|}{ Top 5\% } \\
\hline Gifted $\%$ in Group A & $50.0 \%$ & $55.8 \%$ & $61.5 \%$ & $65.4 \%$ & $69.3 \%$ & $72.0 \%$ & $74.6 \%$ & $76.1 \%$ & $77.3 \%$ & $77.7 \%$ & $78.2 \%$ \\
\hline Gifted $\%$ in Group B & $50.0 \%$ & $44.2 \%$ & $38.5 \%$ & $34.6 \%$ & $30.7 \%$ & $28.0 \%$ & $25.4 \%$ & $23.9 \%$ & $22.7 \%$ & $22.3 \%$ & $21.8 \%$ \\
\hline Average $z$-score for Gifted Group & 1.76 & 1.78 & 1.80 & 1.81 & 1.82 & 1.83 & 1.84 & 1.84 & 1.85 & 1.85 & 1.85 \\
\hline Gifted Group Mean Difference & 0.60 & 0.50 & 0.39 & 0.33 & 0.25 & 0.22 & 0.17 & 0.14 & 0.10 & 0.09 & 0.08 \\
\hline
\end{tabular}


Relative Risk of Identification for Group A
Relative Risk of Identification for Group B

Dissimilarity Index (Gifted/NonGifted)

\begin{tabular}{|c|c|c|c|c|c|c|c|c|c|c|}
\hline 1.00 & 1.26 & 1.60 & 1.89 & 2.26 & 2.58 & 2.94 & 3.18 & 3.41 & 3.49 & 3.59 \\
\hline 1.00 & 0.79 & 0.63 & 0.53 & 0.44 & 0.39 & 0.34 & 0.31 & 0.29 & 0.29 & 0.28 \\
\hline 0.00 & 0.01 & 0.02 & 0.03 & 0.04 & 0.04 & 0.05 & 0.05 & 0.05 & 0.06 & 0.06 \\
\hline $50.0 \%$ & $57.1 \%$ & $63.6 \%$ & $68.3 \%$ & $72.5 \%$ & $75.6 \%$ & $78.2 \%$ & $79.7 \%$ & $80.9 \%$ & $81.2 \%$ & $81.7 \%$ \\
\hline $50.0 \%$ & $42.9 \%$ & $36.4 \%$ & $31.7 \%$ & $27.5 \%$ & $24.4 \%$ & $21.8 \%$ & $20.3 \%$ & $19.1 \%$ & $18.8 \%$ & $18.3 \%$ \\
\hline 2.12 & 2.14 & 2.16 & 2.18 & 2.19 & 2.20 & 2.21 & 2.21 & 2.22 & 2.22 & 2.22 \\
\hline 0.60 & 0.49 & 0.38 & 0.31 & 0.23 & 0.20 & 0.15 & 0.11 & 0.08 & 0.07 & 0.07 \\
\hline 1.00 & 1.33 & 1.75 & 2.15 & 2.63 & 3.09 & 3.59 & 3.92 & 4.23 & 4.32 & 4.48 \\
\hline 1.00 & 0.75 & 0.57 & 0.46 & 0.38 & 0.32 & 0.28 & 0.26 & 0.24 & 0.23 & 0.22 \\
\hline 0.00 & 0.01 & 0.01 & 0.01 & 0.02 & 0.02 & 0.02 & 0.02 & 0.02 & 0.03 & 0.03 \\
\hline $50.0 \%$ & $58.0 \%$ & $65.4 \%$ & $70.6 \%$ & $74.5 \%$ & $78.0 \%$ & $80.5 \%$ & $81.9 \%$ & $82.9 \%$ & $83.4 \%$ & $84.0 \%$ \\
\hline $50.0 \%$ & $42.0 \%$ & $34.6 \%$ & $29.4 \%$ & $25.5 \%$ & $22.0 \%$ & $19.5 \%$ & $18.1 \%$ & $17.1 \%$ & $16.6 \%$ & $16.0 \%$ \\
\hline 2.36 & 2.39 & 2.41 & 2.43 & 2.44 & 2.45 & 2.46 & 2.46 & 2.47 & 2.47 & 2.47 \\
\hline 0.60 & 0.48 & 0.36 & 0.30 & 0.22 & 0.19 & 0.14 & 0.09 & 0.07 & 0.06 & 0.06 \\
\hline 1.00 & 1.38 & 1.89 & 2.39 & 2.93 & 3.53 & 4.13 & 4.54 & 4.85 & 5.01 & 5.25 \\
\hline 1.00 & 0.72 & 0.53 & 0.42 & 0.34 & 0.28 & 0.24 & 0.22 & 0.21 & 0.20 & 0.19 \\
\hline 0.00 & 0.00 & 0.01 & 0.01 & 0.01 & 0.01 & 0.01 & 0.01 & 0.01 & 0.01 & 0.01 \\
\hline
\end{tabular}

Top 2\%

Gifted \% in Group A

Gifted \% in Group B

Average $z$-score for Gifted Group

Gifted Group Mean Difference

Relative Risk of Identification for Group A

Relative Risk of Identification for Group B

Dissimilarity Index (Gifted/Non-

Gifted)

Top $1 \%$

Gifted \% in Group A

Gifted \% in Group B

Average $z$-score for Gifted Group

Gifted Group Mean Difference

Relative Risk of Identification for Group A

Relative Risk of Identification for Group B

Dissimilarity Index (Gifted/Non-

Gifted) 
Table 5

Estimated Gifted Program Data, Under Varying Percentages Selected for Gifted Programs and Levels of District Segregation When d=0.9

\begin{tabular}{|c|c|c|c|c|c|c|c|c|c|c|c|}
\hline & District 1 & District 2 & District 3 & District 4 & District 5 & District 6 & District 7 & District 8 & District 9 & District 10 & District 11 \\
\hline \multicolumn{12}{|l|}{ Top $20 \%$} \\
\hline Gifted \% in Group A & $50.0 \%$ & $55.4 \%$ & $60.8 \%$ & $64.9 \%$ & $68.9 \%$ & $71.8 \%$ & $74.6 \%$ & $76.3 \%$ & $77.9 \%$ & $78.5 \%$ & $78.9 \%$ \\
\hline Gifted \% in Group B & $50.0 \%$ & $44.6 \%$ & $39.2 \%$ & $35.1 \%$ & $31.1 \%$ & $28.2 \%$ & $25.4 \%$ & $23.7 \%$ & $22.1 \%$ & $21.5 \%$ & $21.1 \%$ \\
\hline Average $z$-score for Gifted Group & 0.95 & 0.97 & 1.00 & 1.02 & 1.04 & 1.05 & 1.06 & 1.07 & 1.08 & 1.08 & 1.09 \\
\hline $\begin{array}{l}\text { Gifted Group Mean Difference } \\
\text { Relative Risk of Identification for }\end{array}$ & 0.90 & 0.77 & 0.65 & 0.56 & 0.46 & 0.39 & 0.32 & 0.27 & 0.23 & 0.21 & 0.19 \\
\hline $\begin{array}{l}\text { Group A } \\
\text { Relative Risk of Identification for }\end{array}$ & 1.00 & 1.24 & 1.55 & 1.85 & 2.22 & 2.54 & 2.94 & 3.23 & 3.53 & 3.66 & 3.73 \\
\hline $\begin{array}{l}\text { Group B } \\
\text { Dissimilarity Index (Gifted/Non- }\end{array}$ & 1.00 & 0.81 & 0.65 & 0.54 & 0.45 & 0.39 & 0.34 & 0.31 & 0.28 & 0.27 & 0.27 \\
\hline Gifted) & 0.00 & 0.04 & 0.09 & 0.12 & 0.15 & 0.17 & 0.20 & 0.21 & 0.22 & 0.23 & 0.23 \\
\hline \multicolumn{12}{|l|}{ Top $15 \%$} \\
\hline Gifted $\%$ in Group A & $50.0 \%$ & $56.1 \%$ & $62.2 \%$ & $66.7 \%$ & $71.1 \%$ & $74.0 \%$ & $77.0 \%$ & $78.8 \%$ & $80.4 \%$ & $81.0 \%$ & $81.3 \%$ \\
\hline Gifted \% in Group B & $50.0 \%$ & $43.9 \%$ & $37.8 \%$ & $33.3 \%$ & $28.9 \%$ & $26.0 \%$ & $23.0 \%$ & $21.2 \%$ & $19.6 \%$ & $19.0 \%$ & $18.7 \%$ \\
\hline Average $z$-score for Gifted Group & 1.10 & 1.13 & 1.16 & 1.18 & 1.20 & 1.22 & 1.23 & 1.24 & 1.25 & 1.25 & 1.25 \\
\hline $\begin{array}{l}\text { Gifted Group Mean Difference } \\
\text { Relative Risk of Identification for }\end{array}$ & 0.90 & 0.76 & 0.63 & 0.54 & 0.44 & 0.37 & 0.29 & 0.25 & 0.20 & 0.19 & 0.17 \\
\hline $\begin{array}{l}\text { Group A } \\
\quad \text { Relative Risk of Identification for }\end{array}$ & 1.00 & 1.28 & 1.64 & 2.00 & 2.46 & 2.85 & 3.35 & 3.71 & 4.11 & 4.25 & 4.35 \\
\hline $\begin{array}{l}\text { Group B } \\
\text { Dissimilarity Index (Gifted/Non- }\end{array}$ & 1.00 & 0.78 & 0.61 & 0.50 & 0.41 & 0.35 & 0.30 & 0.27 & 0.24 & 0.24 & 0.23 \\
\hline Gifted) & 0.00 & 0.04 & 0.07 & 0.10 & 0.13 & 0.14 & 0.16 & 0.17 & 0.18 & 0.19 & 0.19 \\
\hline \multicolumn{12}{|l|}{ Top $10 \%$} \\
\hline Gifted $\%$ in Group A & $50.0 \%$ & $57.1 \%$ & $64.3 \%$ & $69.1 \%$ & $73.8 \%$ & $76.7 \%$ & $79.9 \%$ & $81.7 \%$ & $83.3 \%$ & $83.9 \%$ & $84.2 \%$ \\
\hline Gifted \% in Group B & $50.0 \%$ & $42.9 \%$ & $35.7 \%$ & $30.9 \%$ & $26.2 \%$ & $23.3 \%$ & $20.1 \%$ & $18.3 \%$ & $16.7 \%$ & $16.1 \%$ & $15.8 \%$ \\
\hline Average $z$-score for Gifted Group & 1.30 & 1.34 & 1.37 & 1.39 & 1.42 & 1.43 & 1.45 & 1.45 & 1.47 & 1.47 & 1.47 \\
\hline $\begin{array}{l}\text { Gifted Group Mean Difference } \\
\text { Relative Risk of Identification for }\end{array}$ & 0.90 & 0.75 & 0.60 & 0.51 & 0.41 & 0.35 & 0.27 & 0.23 & 0.18 & 0.16 & 0.14 \\
\hline $\begin{array}{l}\text { Group A } \\
\quad \text { Relative Risk of Identification for }\end{array}$ & 1.00 & 1.33 & 1.80 & 2.24 & 2.82 & 3.30 & 3.97 & 4.47 & 4.99 & 5.21 & 5.31 \\
\hline $\begin{array}{l}\text { Group B } \\
\text { Dissimilarity Index (Gifted/Non- }\end{array}$ & 1.00 & 0.75 & 0.56 & 0.45 & 0.35 & 0.30 & 0.25 & 0.22 & 0.20 & 0.19 & 0.19 \\
\hline Gifted) & 0.00 & 0.03 & 0.06 & 0.08 & 0.10 & 0.11 & 0.12 & 0.13 & 0.13 & 0.14 & 0.14 \\
\hline \multicolumn{12}{|l|}{ Top 5\% } \\
\hline Gifted \% in Group A & $50.0 \%$ & $58.8 \%$ & $67.7 \%$ & $72.9 \%$ & $77.9 \%$ & $80.8 \%$ & $83.9 \%$ & $85.6 \%$ & $87.1 \%$ & $87.6 \%$ & $87.7 \%$ \\
\hline Gifted \% in Group B & $50.0 \%$ & $41.2 \%$ & $32.3 \%$ & $27.1 \%$ & $22.1 \%$ & $19.2 \%$ & $16.1 \%$ & $14.4 \%$ & $12.9 \%$ & $12.4 \%$ & $12.3 \%$ \\
\hline Average $z$-score for Gifted Group & 1.61 & 1.65 & 1.69 & 1.72 & 1.74 & 1.76 & 1.77 & 1.78 & 1.80 & 1.80 & 1.80 \\
\hline Gifted Group Mean Difference & 0.90 & 0.72 & 0.56 & 0.47 & 0.37 & 0.31 & 0.23 & 0.20 & 0.15 & 0.13 & 0.12 \\
\hline
\end{tabular}


Relative Risk of Identification for Group A

Relative Risk of Identification for Group B

Dissimilarity Index (Gifted/NonGifted)

\begin{tabular}{|c|c|c|c|c|c|c|c|c|c|c|}
\hline 1.00 & 1.43 & 2.10 & 2.70 & 3.53 & 4.21 & 5.22 & 5.96 & 6.74 & 7.05 & 7.12 \\
\hline 1.00 & 0.70 & 0.48 & 0.37 & 0.28 & 0.24 & 0.19 & 0.17 & 0.15 & 0.14 & 0.14 \\
\hline 0.00 & 0.02 & 0.04 & 0.05 & 0.06 & 0.06 & 0.07 & 0.07 & 0.07 & 0.08 & 0.08 \\
\hline $50.0 \%$ & $60.9 \%$ & $72.0 \%$ & $77.6 \%$ & $82.3 \%$ & $85.2 \%$ & $87.8 \%$ & $89.3 \%$ & $90.4 \%$ & $90.9 \%$ & $91.1 \%$ \\
\hline $50.0 \%$ & $39.1 \%$ & $28.0 \%$ & $22.4 \%$ & $17.7 \%$ & $14.8 \%$ & $12.2 \%$ & $10.7 \%$ & $9.6 \%$ & $9.1 \%$ & $8.9 \%$ \\
\hline 1.97 & 2.02 & 2.06 & 2.09 & 2.12 & 2.14 & 2.15 & 2.16 & 2.18 & 2.18 & 2.18 \\
\hline 0.90 & 0.70 & 0.50 & 0.43 & 0.32 & 0.28 & 0.21 & 0.17 & 0.12 & 0.11 & 0.09 \\
\hline 1.00 & 1.56 & 2.56 & 3.46 & 4.65 & 5.74 & 7.17 & 8.32 & 9.45 & 9.97 & 10.22 \\
\hline 1.00 & 0.64 & 0.39 & 0.29 & 0.21 & 0.17 & 0.14 & 0.12 & 0.11 & 0.10 & 0.10 \\
\hline 0.00 & 0.01 & 0.02 & 0.02 & 0.03 & 0.03 & 0.03 & 0.03 & 0.03 & 0.03 & 0.03 \\
\hline $50.0 \%$ & $62.3 \%$ & $74.8 \%$ & $80.5 \%$ & $84.9 \%$ & $87.5 \%$ & $90.0 \%$ & $91.3 \%$ & $92.2 \%$ & $92.5 \%$ & $93.0 \%$ \\
\hline $50.0 \%$ & $37.7 \%$ & $25.2 \%$ & $19.5 \%$ & $15.1 \%$ & $12.5 \%$ & $10.0 \%$ & $8.7 \%$ & $7.8 \%$ & $7.5 \%$ & $7.0 \%$ \\
\hline 2.21 & 2.27 & 2.32 & 2.35 & 2.38 & 2.40 & 2.41 & 2.42 & 2.44 & 2.44 & 2.44 \\
\hline 0.90 & 0.68 & 0.47 & 0.40 & 0.30 & 0.26 & 0.19 & 0.14 & 0.11 & 0.09 & 0.07 \\
\hline 1.00 & 1.65 & 2.96 & 4.14 & 5.62 & 7.00 & 8.98 & 10.56 & 11.83 & 12.40 & 13.20 \\
\hline 1.00 & 0.60 & 0.34 & 0.24 & 0.18 & 0.14 & 0.11 & 0.09 & 0.08 & 0.08 & 0.08 \\
\hline 0.00 & 0.00 & 0.01 & 0.01 & 0.01 & 0.02 & 0.02 & 0.02 & 0.02 & 0.02 & 0.02 \\
\hline
\end{tabular}

Top 2\%

Gifted \% in Group A

Gifted \% in Group B

Average $z$-score for Gifted Group

Gifted Group Mean Difference

Relative Risk of Identification for Group A

Relative Risk of Identification for Group B

Dissimilarity Index (Gifted/Non-

Gifted)

Top $1 \%$

Gifted \% in Group A

Gifted \% in Group B

Average $z$-score for Gifted Group

Gifted Group Mean Difference

Relative Risk of Identification for Group A

Relative Risk of Identification for Group B

Dissimilarity Index (Gifted/Non-

Gifted) 
Table 6

Estimated Gifted Program Data, Under Varying Percentages Selected for Gifted Programs and Levels of District Segregation When d $=1.2$

\begin{tabular}{|c|c|c|c|c|c|c|c|c|c|c|c|}
\hline & District 1 & District 2 & District 3 & District 4 & District 5 & District 6 & District 7 & District 8 & District 9 & District 10 & District 11 \\
\hline \multicolumn{12}{|l|}{ Top $20 \%$} \\
\hline Gifted \% in Group A & $50.0 \%$ & $56.9 \%$ & $63.9 \%$ & $69.1 \%$ & $74.2 \%$ & $77.7 \%$ & $81.2 \%$ & $83.0 \%$ & $85.0 \%$ & $85.7 \%$ & $86.3 \%$ \\
\hline Gifted \% in Group B & $50.0 \%$ & $43.1 \%$ & $36.1 \%$ & $30.9 \%$ & $25.8 \%$ & $22.3 \%$ & $18.8 \%$ & $17.0 \%$ & $15.0 \%$ & $14.3 \%$ & $13.7 \%$ \\
\hline Average $z$-score for Gifted Group & 0.80 & 0.84 & 0.89 & 0.92 & 0.95 & 0.98 & 1.00 & 1.01 & 1.03 & 1.03 & 1.03 \\
\hline $\begin{array}{l}\text { Gifted Group Mean Difference } \\
\text { Relative Risk of Identification for }\end{array}$ & 1.20 & 1.02 & 0.86 & 0.74 & 0.62 & 0.53 & 0.43 & 0.37 & 0.30 & 0.27 & 0.25 \\
\hline $\begin{array}{l}\text { Group A } \\
\text { Relative Risk of Identification for }\end{array}$ & 1.00 & 1.32 & 1.77 & 2.23 & 2.87 & 3.48 & 4.32 & 4.88 & 5.65 & 5.99 & 6.27 \\
\hline $\begin{array}{l}\text { Group B } \\
\text { Dissimilarity Index (Gifted/Non- }\end{array}$ & 1.00 & 0.76 & 0.57 & 0.45 & 0.35 & 0.29 & 0.23 & 0.20 & 0.18 & 0.17 & 0.16 \\
\hline Gifted) & 0.00 & 0.06 & 0.11 & 0.15 & 0.19 & 0.22 & 0.25 & 0.26 & 0.28 & 0.29 & 0.29 \\
\hline \multicolumn{12}{|l|}{ Top $15 \%$} \\
\hline Gifted $\%$ in Group A & $50.0 \%$ & $58.0 \%$ & $66.0 \%$ & $71.6 \%$ & $77.0 \%$ & $80.4 \%$ & $84.0 \%$ & $85.6 \%$ & $87.5 \%$ & $88.1 \%$ & $88.6 \%$ \\
\hline Gifted \% in Group B & $50.0 \%$ & $42.0 \%$ & $34.0 \%$ & $28.4 \%$ & $23.0 \%$ & $19.6 \%$ & $16.0 \%$ & $14.4 \%$ & $12.5 \%$ & $11.9 \%$ & $11.4 \%$ \\
\hline Average $z$-score for Gifted Group & 0.95 & 1.00 & 1.05 & 1.09 & 1.13 & 1.15 & 1.17 & 1.19 & 1.20 & 1.21 & 1.21 \\
\hline $\begin{array}{l}\text { Gifted Group Mean Difference } \\
\text { Relative Risk of Identification for }\end{array}$ & 1.20 & 1.00 & 0.82 & 0.71 & 0.58 & 0.50 & 0.39 & 0.34 & 0.27 & 0.24 & 0.22 \\
\hline $\begin{array}{l}\text { Group A } \\
\text { Relative Risk of Identification for }\end{array}$ & 1.00 & 1.38 & 1.94 & 2.51 & 3.34 & 4.11 & 5.24 & 5.95 & 7.00 & 7.43 & 7.80 \\
\hline $\begin{array}{l}\text { Group B } \\
\text { Dissimilarity Index (Gifted/Non- }\end{array}$ & 1.00 & 0.72 & 0.51 & 0.40 & 0.30 & 0.24 & 0.19 & 0.17 & 0.14 & 0.13 & 0.13 \\
\hline Gifted) & 0.00 & 0.05 & 0.10 & 0.13 & 0.16 & 0.18 & 0.20 & 0.21 & 0.23 & 0.23 & 0.23 \\
\hline \multicolumn{12}{|l|}{ Top $10 \%$} \\
\hline Gifted $\%$ in Group A & $50.0 \%$ & $59.5 \%$ & $69.0 \%$ & $74.8 \%$ & $80.4 \%$ & $83.7 \%$ & $87.1 \%$ & $88.6 \%$ & $90.3 \%$ & $90.7 \%$ & $91.2 \%$ \\
\hline Gifted $\%$ in Group B & $50.0 \%$ & $40.5 \%$ & $31.0 \%$ & $25.2 \%$ & $19.6 \%$ & $16.3 \%$ & $12.9 \%$ & $11.4 \%$ & $9.7 \%$ & $9.3 \%$ & $8.8 \%$ \\
\hline Average $z$-score for Gifted Group & 1.15 & 1.21 & 1.27 & 1.31 & 1.35 & 1.37 & 1.40 & 1.41 & 1.42 & 1.43 & 1.43 \\
\hline $\begin{array}{l}\text { Gifted Group Mean Difference } \\
\text { Relative Risk of Identification for }\end{array}$ & 1.20 & 0.98 & 0.77 & 0.66 & 0.53 & 0.45 & 0.35 & 0.30 & 0.23 & 0.21 & 0.19 \\
\hline $\begin{array}{l}\text { Group A } \\
\text { Relative Risk of Identification for }\end{array}$ & 1.00 & 1.47 & 2.23 & 2.97 & 4.10 & 5.15 & 6.75 & 7.80 & 9.28 & 9.77 & 10.33 \\
\hline $\begin{array}{l}\text { Group B } \\
\text { Dissimilarity Index (Gifted/Non- }\end{array}$ & 1.00 & 0.68 & 0.45 & 0.34 & 0.24 & 0.19 & 0.15 & 0.13 & 0.11 & 0.10 & 0.10 \\
\hline Gifted) & 0.00 & 0.04 & 0.08 & 0.10 & 0.12 & 0.14 & 0.15 & 0.15 & 0.16 & 0.16 & 0.16 \\
\hline \multicolumn{12}{|l|}{ Top 5\% } \\
\hline Gifted \% in Group A & $50.0 \%$ & $61.9 \%$ & $74.1 \%$ & $79.7 \%$ & $85.4 \%$ & $88.1 \%$ & $91.0 \%$ & $92.1 \%$ & $93.4 \%$ & $93.7 \%$ & $94.1 \%$ \\
\hline Gifted $\%$ in Group B & $50.0 \%$ & $38.1 \%$ & $25.9 \%$ & $20.3 \%$ & $14.6 \%$ & $11.9 \%$ & $9.0 \%$ & $7.9 \%$ & $6.6 \%$ & $6.3 \%$ & $5.9 \%$ \\
\hline Average $z$-score for Gifted Group & 1.46 & 1.53 & 1.60 & 1.64 & 1.69 & 1.71 & 1.74 & 1.75 & 1.77 & 1.77 & 1.78 \\
\hline Gifted Group Mean Difference & 1.20 & 0.95 & 0.69 & 0.60 & 0.46 & 0.40 & 0.30 & 0.25 & 0.19 & 0.17 & 0.15 \\
\hline
\end{tabular}


Relative Risk of Identification for Group A

Relative Risk of Identification for Group B

Dissimilarity Index (Gifted/NonGifted)

\begin{tabular}{|c|c|c|c|c|c|c|c|c|c|c|}
\hline 1.00 & 1.63 & 2.86 & 3.93 & 5.87 & 7.41 & 10.12 & 11.73 & 14.08 & 14.78 & 16.06 \\
\hline 1.00 & 0.61 & 0.35 & 0.25 & 0.17 & 0.13 & 0.10 & 0.09 & 0.07 & 0.07 & 0.06 \\
\hline 0.00 & 0.02 & 0.05 & 0.06 & 0.07 & 0.08 & 0.08 & 0.08 & 0.09 & 0.09 & 0.09 \\
\hline $50.0 \%$ & $64.9 \%$ & $80.0 \%$ & $84.9 \%$ & $90.0 \%$ & $92.0 \%$ & $94.3 \%$ & $95.0 \%$ & $95.8 \%$ & $95.9 \%$ & $96.4 \%$ \\
\hline $50.0 \%$ & $35.1 \%$ & $20.0 \%$ & $15.1 \%$ & $10.0 \%$ & $8.0 \%$ & $5.7 \%$ & $5.0 \%$ & $4.2 \%$ & $4.1 \%$ & $3.6 \%$ \\
\hline 1.82 & 1.91 & 1.99 & 2.03 & 2.08 & 2.11 & 2.13 & 2.14 & 2.16 & 2.16 & 2.17 \\
\hline 1.20 & 0.93 & 0.60 & 0.53 & 0.40 & 0.34 & 0.26 & 0.21 & 0.15 & 0.13 & 0.13 \\
\hline 1.00 & 1.85 & 4.00 & 5.61 & 9.04 & 11.56 & 16.45 & 18.86 & 22.55 & 23.37 & 26.49 \\
\hline 1.00 & 0.54 & 0.25 & 0.18 & 0.11 & 0.09 & 0.06 & 0.05 & 0.04 & 0.04 & 0.04 \\
\hline 0.00 & 0.01 & 0.02 & 0.03 & 0.03 & 0.03 & 0.04 & 0.04 & 0.04 & 0.04 & 0.04 \\
\hline $50.0 \%$ & $66.8 \%$ & $84.0 \%$ & $88.0 \%$ & $92.6 \%$ & $94.2 \%$ & $95.9 \%$ & $96.2 \%$ & $96.8 \%$ & $97.1 \%$ & $97.5 \%$ \\
\hline $50.0 \%$ & $33.2 \%$ & $16.0 \%$ & $12.0 \%$ & $7.4 \%$ & $5.8 \%$ & $4.1 \%$ & $3.8 \%$ & $3.2 \%$ & $2.9 \%$ & $2.5 \%$ \\
\hline 2.07 & 2.16 & 2.26 & 2.30 & 2.35 & 2.37 & 2.40 & 2.41 & 2.42 & 2.43 & 2.43 \\
\hline 1.20 & 0.92 & 0.54 & 0.49 & 0.36 & 0.30 & 0.23 & 0.19 & 0.15 & 0.10 & 0.12 \\
\hline 1.00 & 2.02 & 5.23 & 7.33 & 12.59 & 16.18 & 23.16 & 25.50 & 30.11 & 33.35 & 38.25 \\
\hline 1.00 & 0.50 & 0.19 & 0.14 & 0.08 & 0.06 & 0.04 & 0.04 & 0.03 & 0.03 & 0.03 \\
\hline 0.00 & 0.01 & 0.01 & 0.02 & 0.02 & 0.02 & 0.02 & 0.02 & 0.02 & 0.02 & 0.02 \\
\hline
\end{tabular}

Top 2\%

Gifted \% in Group A

Gifted \% in Group B

Average $z$-score for Gifted Group

Gifted Group Mean Difference

Relative Risk of Identification for Group A

Relative Risk of Identification for

Group B

Dissimilarity Index (Gifted/Non-

Gifted)

Top $1 \%$

Gifted \% in Group A

Gifted \% in Group B

Average $z$-score for Gifted Group

Gifted Group Mean Difference

Relative Risk of Identification for Group A

Relative Risk of Identification for Group B

Dissimilarity Index (Gifted/Non- 
The tables illustrate several of the consequences of using building-level local norms to select students for gifted programs. First, there is an inverse relationship between district integration and gifted program integration. In other words, building-level local norms produce the most diverse, racially integrated gifted programs in the most segregated districts — and vice versa. As long as the achievement gap was greater than zero (and regardless of the cutoff score), only the fully segregated school district (District 1) produced a gifted program that was representative of the district's general student population. Conversely, the most racially integrated district (District 11) always had the gifted program with the lowest diversity and highest dissimilarity index (when the achievement gap was held constant). ${ }^{1}$ Additionally, the relative risks of being selected for a gifted program for the two groups diverge as a district became more integrated or in districts with higher achievement gaps, with students from Group A having higher relative risks of being selected for the gifted program and members of Group B successively lower relative risks in more integrated districts and in scenarios where achievement gaps are larger. Indeed, the only time when members of the lower scoring group had the same relative risk of being identified as gifted as members of the higher scoring group was in the fully segregated District 1 (as shown by the relative risk values of 1.00 in Tables 2-6) or when there was no mean difference in scores across groups (shown in Table 2). These tendencies were

\footnotetext{
${ }^{1}$ The highest gifted/non-gifted dissimilarity index in Tables 2-6 is 0.29 , and most are below 0.10 . At first glance, this may seem like a positive development indicating that the gifted programs are less segregated than the general population in most simulated districts. However, this a mathematical artifact of the way the dissimilarity index is calculated. This is because dissimilarity index is a proportion of students from a demographic who would have to move schools (in the district dissimilarity indexes) or change identification status (in the gifted programs' dissimilarity indexes). Because gifted students are a relatively small percentage of the district population (1-20\% in Tables 2-6), the gifted/non-gifted dissimilarity index requires fewer students to change in order to integrate a smaller program. Therefore, the dissimilarity indexes for the districts and the dissimilarity indexes for the gifted programs are not directly comparable.
} 
apparent, regardless of the percentage of students selected for a gifted program within each school.

Building-level local norms also impacted the academic composition of the gifted group. For all cutoffs, the fully segregated district had the lowest average $z$-score for students admitted to the gifted program, and this threshold increased as districts became more integrated. Among students selected for the gifted program, average demographic group differences in academic achievement were always largest in the fully segregated district, while the fully integrated district had the smallest differences in academic achievement between gifted children from different racial groups. Thus, highly segregated districts produce a gifted program that displays large average group differences in ability within the gifted population, and a highly integrated district produces a more academically homogeneous gifted group (except when the achievement gap was $d=0$ ). But this academic homogeneity within gifted programs occurs in tandem with demographic homogeneity within those programs when there is an achievement gap present in the general district population.

\section{Discussion}

\section{Inverse Relationship Between School and Gifted Program Integration}

These results show why local norms are only partially effective in diversifying gifted programs: these is an inverse relationship between school-level integration and gifted program integration when the same percentage of students are selected from each school to participate in a gifted program. As schools become more integrated, the ability for local norms to select a representative sample of gifted students becomes more limited (unless there is no achievement gap between groups). This presents a conundrum for advocates of diversity in education. Local building-level norms trade one form of segregation for another. Assuming one selects the same 
percentage of students from each building and applies the same cutoff to all demographic groups within a school, a school district can have integrated schools or an integrated gifted programbut not both simultaneously. The exception to this rule occurs when there is no mean difference in scores across groups, as shown in Table 2. The relationship between district segregation and gifted program segregation will be most noticeable in districts with large achievement gaps between groups.

Moreover, school-level integration and the use of building norms have consequences on the population selected for the gifted program. When the dissimilarity index was equal to 1.0 (which indicates completely segregated schools), the group differences in ability within the gifted program was equal to the average group difference in the general population. This occurred because selecting children from completely segregated schools is functionally equivalent to using race-specific norms or racial quotas that force the gifted program to be representative group of the general district (thereby preserving mean differences seen in the general student population). Conversely, when the dissimilarity index was zero in a fully integrated district, the mean group differences within the gifted population were smallest; as the average group difference in scores increased, the fully integrated district's gifted program dissimilarity index increased. This is because in a perfectly integrated school district, each school serves as a representative sample of the entire district, and school-level norms replicate the results that would occur if district-level norms were used.

\section{Consequences for Gifted Program Implementation}

The inverse relationship between district and gifted program integration has important implications for gifted programming. For example, if each school creates its own self-contained gifted program (e.g., an enrichment program), then what selected students are ready to learn may 
vary across schools. Regardless of the segregation level of the schools within a district, children who are labeled as gifted at schools with lower average levels of achievement will be (on average) prepared for curriculum that is less advanced than what gifted children at schools with higher levels of average achievement are prepared for. Critics of gifted education or district policy may see this as perpetuating another form of injustice-especially in a highly segregated district, which will have more students from a lower scoring group experiencing a less challenging gifted program.

On the other hand, customizing gifted programs for different school populations is faithful to the principle that gifted programs are appropriate for children who academically outperform their peers; the fact that a child at a low performing school may not qualify for a gifted program designed for a school with a higher average level of achievement does not abrogate the discrepancy between that child's abilities and the typical learning environment in their current school. Local, building-level gifted programs may also be better at serving children whose parents do not have the resources to send their child to a centralized gifted program (e.g., a magnet school). There are advantages to bringing a gifted services to children in the school buildings they already attend, even if services are not uniform across schools.

Standardizing gifted programming across schools or sending all gifted children to the same program (e.g., a magnet school) can create problems. If students are selected for a common program under heterogeneous admissions criteria (as in the more segregated districts in these calculations), then placing them in a common gifted program will reproduce some of the average differences of academic achievement that are seen in the general district population, thereby increasing the likelihood that one program will not be capable of serving all students labeled as gifted. Tables 3-6 show that these mean group differences within a gifted program are most 
severe in highly segregated districts but decrease in magnitude in more integrated districts. However, this narrowing of academic differences within the gifted population comes at a cost of admitting fewer children from the lower scoring group into the common gifted program, resulting in a gifted program that is unrepresentative of the general district population.

When considering the achievement gaps within the gifted program shown in Tables 3-6, it is important to remember the individual differences — not just average group differenceswithin the gifted population. There is as much range in ability in the top 5\% of a normal distribution as there is in the middle 90\% (Rambo-Hernandez \& Warne, 2015), and the research from the Study of Mathematically Precocious Youth and similar samples shows that even within the top $1 \%$ of ability there are noticeable differences in accomplishment (Makel et al., 2016). Identifying a cutoff that produces small mean differences between demographic groups will still result in a gifted program that contains a great deal of individual academic diversity. Therefore, a variety of gifted services will be appropriate for most gifted populations, though this is especially true for districts with larger percentages of students labeled as gifted and which have large average differences between demographic groups within the gifted population.

\section{Disproportionality as a Consequence of the Achievement Gap}

It is important to recognize that these results arise from three parameters: the size of the average group difference in abilities, the degree of school district integration, and the percentage of students from each school selected for a gifted program. The results in our simulation—some of which may appear unfair or socially regressive to some readers — did not arise from any discriminatory process. This is purely a mathematical phenomenon that occurs any time an average difference in scores appears across two or more groups and a uniform selection cutoff is applied (Petersen \& Novick, 1976). Therefore, when achievement gaps are present among 
groups, common proposals for remedying disproportionate representation of gifted programs, such as placing greater value on cultures of diverse gifted children (Ford, 2014), increasing the diversity of school personnel (Grissom \& Redding, 2016), and using nonverbal tests for gifted identification (Naglieri \& Ford, 2003) will have — at best - modest impacts on diversity in gifted programs. These policies will have little effect because they do not meaningfully change the major proximal cause of disproportionate representation: the achievement gap (Warne, in press; Worrell et al., 2019).

Likewise, because disproportionate representation in gifted programs can arise as a mathematical consequence of achievement gaps, a gifted program that does not reflect the demographics of a district's general population is not ipso facto evidence of discrimination in the selection process for gifted programs. Thus, simplistic statistical cutoffs to judge whether programs are sufficiently diverse may present an inaccurate view of whether the identification process is discriminatory. For example, Yoon and Gentry (2009) examined the proportion of students identified as gifted in different racial groups and judged that a state had proportionate representation for a group if the group's proportion of students labeled as gifted was statistically equal to its proportion in the general student population (two-tailed $p>.05$ ). When states did not meet this threshold, the authors suggested that the results may be because "identification procedures may not be totally free of biases" (Yoon \& Gentry, 2009, p. 132). As another example, Ford (2014) proposed an Equity Index that would serve as a minimum desirable threshold of a group's representation in a gifted program. If a gifted program has a student group that is less than $80 \%$ of a group's proportion ${ }^{2}$ in the general district population, then this

\footnotetext{
${ }^{2}$ Ford derived this threshold from the definition of "adverse impact" set by the Office for Civil Rights for employment decisions. It is an arbitrary threshold established by bureaucrats without scientific, statistical, or logical basis. It is not clear why a rule designed in employment law for adults should apply unchanged to children in the K12 education system.
} 
underrepresentation "is beyond statistical chance; thus, human error is operating" (Ford, 2014, p. 145). But the calculations in this article show that violating either standard is not a guarantee that discrimination or human error has happened. Ironically, both standards can punish districts that have taken strides towards integrating the general student population. This may have been one of the reasons that $71.6 \%$ of Texas school districts - in a southern state with strong pressures to desegregate schools within districts - had a lower percentage of Hispanic students than allowed by Ford's (2014) Equity Index (Lamb et al., 2019). This is not to say that discriminatory identification procedures do not happen. Rather, we believe that underrepresentation is a complex issue (Worrell et al., 2019) that cannot be well represented with simplistic metrics. Regression analysis of real data support the results of this simulation study. Statistically controlling for achievement differences among demographic groups makes underrepresentation of African American and Hispanic students in gifted programs sometimes disappears (e.g., Grissom \& Redding, 2016; Warne et al., 2013). This supports claims (e.g., from Worrell et al., 2019) that the achievement gap (or its cause, or something highly correlated with its cause) is one of the major underlying causes of disproportionate representation of racial and ethnic groups in gifted programs.

\section{Recommendations}

For scholars. The trade-off between district integration and gifted program diversity may be dismaying for many gifted education scholars and practitioners. However, there are recommendations for how the field can accommodate the mathematical expectations that the simulation produces.

First, gifted education scholars should not assume that disproportionate representation is - by itself — evidence of discrimination in the gifted identification process. Instead, 
disproportionate representation should be a sign that the possibility of discrimination has occurred; identifying a lack of diversity in a gifted program is the first step to discovering whether discriminatory processes are in place — not a last step. Tables 3-6 show that even appalling levels of disproportionate representation can be merely the consequence of an achievement gap in highly integrated schools. This is especially true when achievement gaps are large and/or the percentage of students from each school selected for a gifted program is small.

This leads to our second recommendation for gifted education scholars: when discussing disproportionate representation in gifted programs, it should be standard practice to control for pre-existing differences in academic achievement and/or related variables (e.g., aptitude test scores) that are used in the gifted identification process. Because disproportionate representation is a mathematical consequence of applying uniform selection rules when group differences exist (except in perfectly segregated districts), these average score differences must be controlled prior to investigating any other theorized causes for under- or overrepresentation of demographic groups in gifted programs. Failure to do so will produce distorted results about the apparent effect of other posited causes of disproportionate representation.

For practitioners, in the long-term. For practitioners, the main takeaway from this article is the importance of addressing the achievement gap among demographic groups. As prior researchers have stated,

... there is an expectation that the demographic profile of gifted students should mirror the demographic profile of the school or school district ... Although this expectation is an important aspirational goal for the field, it cannot be met using the same cutoff scores or identification protocols until achievement gaps are eliminated, an outcome that will require ongoing and sustained efforts. (Worrell et al., 2019, p. 565) 
Because of the results of the simulation and the pervasive reality of achievement gaps, we propose that the most effective method of creating gifted programs that reflect the demographics of the general student population is to close the achievement gap among groups because this will address a likely proximal cause of the demographic imbalance seen in so many gifted programs (Warne, in press; Worrell \& Dixson, 2018). Other (well-intentioned) proposals that ignore the achievement gap are not promising leads for solving this problem. Instead, projects to improve the achievement of underrepresented gifted children in order to diversity gifted programs have shown much more favorable results (e.g., Olszewski-Kubilius et al., 2017; Swanson, 2006).

Closing the achievement gap is easier said than done. These average differences in academic performance among groups develop early in life (Curran \& Kellogg, 2016; Little, 2017; Reardon \& Portilla, 2016) and are still present at the end of students' K-12 experience when many students take college admissions tests (Warne, 2020). The good news is that there has been progress in recent decades in reducing the average achievement test score differences among racial/ethnic groups within the United States (de Brey et al., 2019), though some scholars have presented data that this change in score gaps has stagnated (e.g., Murray, 2021). Gifted education personnel who aim to increase the diversity of their gifted programs should be part of the efforts to reduce the achievement gap among demographic groups because solving the achievement gap problem will likely do much to solve the problem of disproportionate representation of gifted programs. Indeed, this simulation and studies of real data (Carman, 2018, 2020; Lakin, 2018; Peters et al., 2019) show that two strategies can both improve diversity of gifted programs and help narrow the achievement gap: (1) identifying children through local, building-level norms, and (2) lowering the threshold for admission into gifted programs. Both of these strategies identify more children who are above average scholastically but less 
academically elite than a gifted group selected with district-level norms. If the gifted programs that these children attend are effective at increasing academic achievement, then using buildinglevel norms and then giving selected children an effective gifted program can help narrow the achievement gap between demographic groups.

For practitioners, in the short-term. Closing the achievement gap is a slow process, and many people with the goal of achieving proportionate representation may not want (or be able to) wait years or decades for the changes to occur that will close the achievement gapassuming that societal trends and implemented policies will close it at all. Gifted education personnel may be under pressure to increase diversity in gifted programs immediately. Because closing the achievement gap does not occur quickly, short-term solutions do not solve this fundamental cause of disproportionate representation in gifted programs. Instead, short-term adjustments to the gifted identification process involve trade-offs.

Our first suggestion is to use building-level norms instead of district- or national-level norms when selecting children for a gifted program. In most cases, building norms will produce more diverse gifted programs than national or district norms will. This is apparent when comparing results for a table's District 11 (the perfectly integrated district, which is the equivalent of selecting children with district-level norms) with other results in the same table. Although studies with real data (Carman et al., 2018, 2020; Peters et al., 2019) show that building-level norms will not produce gifted programs that have perfectly proportional representation, they are better than norms based on the population in an entire district or the nation, even if (in most cases) these building-level norms do not result in perfect proportionality in a gifted program. 
Another option is to use measures that have smaller differences between group averages. For example, academic achievement tests tend to have smaller mean score differences across racial groups than aptitude tests do (Warne, 2020). Smaller group differences will reduce the disparity in the percentages of students who meet or exceed a cutoff to be labeled as gifted, resulting in programs that better reflect the demographics of the general student body, as is clear when comparing corresponding cells in Tables 2-6 or Tables S1-21. However, this option runs into a fundamental difficulty: tests that tap general, abstract abilities most strongly associated with intellectual giftedness tend to have larger the score differences among racial groups; tests with narrower gaps among racial groups tend to be poor measures of general intellectual ability (Warne, 2016b). This phenomenon is called Spearman's hypothesis and is well documented in the intelligence literature (e.g., Jensen, 1980, 1985, 1998; te Nijenhuis et al., 2015; Warne, 2016b, 2020) but is rarely discussed in gifted education publications.

Another possibility for increasing the representation of gifted programs: lowering the standards for admission. Tables 3-6 show that as gifted programs became less elite, the demographic of the students admitted to the program was closer to the 50-50 split in the general district population. This is because the proportion of a group that exceeds increasing cutoffs gets smaller more rapidly for a group with a lower average than a group with a higher average, thus resulting in a greater demographic imbalanced as cutoffs increase (Warne, 2020). This relationship between cutoff score selectivity and diversity of the group exceeding the cutoff is not merely theoretical; it is in accordance with studies of real data examining gifted group diversity under different cutoff conditions (e.g., Carman et al., 2018, 2020; Lakin, 2018).

The difficulty with this option is that it can lower the threshold for giftedness so much that the label becomes meaningless. For example, to meet Ford's (2014) Equity Index, a district 
with a dissimilarity index of 0.75 (on par with the most segregated districts in the United States) would have to admit the top $24 \%$ of students from each school. A district with a dissimilarity index of 0.25 would need to admit the top $66 \%$ of students from each school to the gifted program, and a perfectly integrated district would be required to label the top $71 \%$ of students as gifted. ${ }^{3}$ While an operational definition of "giftedness" as being equivalent to the top $24 \%$ of students in achievement may be justifiable, in highly integrated districts, stating that two-thirds or more children are "gifted" is much more difficult to justify if the term "gifted" is going to retain any of its connections with high scholastic performance. ${ }^{4}$ This expansion of the "gifted" label to the point where it loses any meaning is not a mere theoretical prospect. Recently, the public schools in Charlottesville, Virginia, announced (Knott, 2021) that —after a push to improve the representativeness of the demographics of the district's gifted program - that $86 \%$ of students in Grades 3-11 were labeled as "gifted." Equity achieved!

The average group differences in a district also strongly influence its ability to meet Ford's (2014) standards for her Equity Index. When there are no average group differences (i.e., $d=0$; see Table 2), every district—regardless of integration level—can meet this standard. When $d=.30$ (in Table 3 ), this standard is only consistently met when selecting the top $20 \%$ from schools for a gifted program (or top $15 \%$ for more segregated districts). With larger mean group

\footnotetext{
${ }^{3}$ These percentages were found by assuming that two groups had a mean difference of $d=.88$, which is the nationwide average difference in math and reading achievement between White and Black students within the United States (Lipsey et al., 2012, p. 30). Successive cutoffs were examined for each dissimilarity index, and the maximum cutoff was found that would meet the standards of the Equity Index. The percentage of all students within a district who exceeded this maximum cutoff is then reported in the paragraph.

${ }^{4}$ Applying Ford's (2014) Equity Index to intelligence tests — which tend to have larger score gaps than academic achievement tests-requires lowering the threshold for "giftedness" even further to an IQ of 76 for two groups with averages that are $d=1.00$ apart (Warne, 2020, pp. 325-326). Applying this cutoff would mean that the top $94.5 \%$ of the population would be labeled as "gifted." With an IQ of 70-75 being the approximate threshold for a person to be a candidate to be diagnosed with an intellectual disability, this rule would mean that almost everybody who does not have an intellectual disability would qualify as "gifted." This is not a viable operationalization of the "gifted" label.
} 
differences (and more elite groups identified as gifted), it is nearly impossible to meet the standards of Ford's Equity Index while also holding all students to the same admission standard.

Much less drastic in its consequences would be selectively lowering the cutoff for some demographic groups with lower score averages and/or raising cutoffs for groups with higher averages. These cutoff adjustments for different racial groups can occur directly or indirectly. Direct cutoff adjustments are policies that explicitly set varying cutoffs (or are mathematical equivalents to setting varying cutoffs) in order to achieve a desired level of diversity in a gifted program. A quota system, where individuals within a racial group are selected until the exact diversity aims are achieved, is one method of explicitly varying cutoffs. This is becauseassuming students are selected within groups on the basis of their academic performancegroups with higher average scores will fill their quota with students who are more academically elite, whereas a group with a lower average score will exhaust its supply of elite candidates and have to admit students who are closer to average. A mathematically equivalent system to quotas is race norming, which is the process of creating separate norm groups for different races and only comparing people to their racial group's norms and admitting them to the gifted program at proportional rates.

Both quotas and race norming force a selected group to be representative of the general population, as shown both theoretically (Petersen \& Novick, 1976) and with empirical data (e.g., Carman et al., 2018, 2020). Selecting the same percentage of students from schools in a district consisting of perfectly segregated schools (i.e., the first column of numbers in Tables 2-6 and Tables S1-S21) is the equivalent of race norming because students were only compared to others of their same race. The result is a gifted program that perfectly reflected the overall district demographics. The disadvantage of these methods is in their legality; quotas in education are 
illegal in the United States (Sander \& Taylor, 2012). Race norming is illegal in hiring and promoting (Calvin, 2000) and often challenged in the courts when it occurs in education (Sander $\&$ Taylor, 2012). Some states have also passed laws banning the consideration of race when making educational decisions, and these laws may apply gifted education programs in public schools.

Another direct method of adjusting cutoffs for different demographic groups is to create a system that awards bonus points to members of underrepresented groups and/or penalizes students who belong to groups that are overrepresented in gifted programs. These adjustments to the cutoff are not always based on empirical considerations and can take a variety of forms, depending on the groups benefited or penalized and the magnitude of the adjustment(s). Unlike quotas and race norming, though, cutoff adjustments are not guaranteed to produce a gifted program with demographics that mirrors the general student population. Carman et al. (2018, 2020) investigated the cutoff adjustment based on a regression model where demographic variables were regressed onto aptitude test scores. The unstandardized regression weight for a demographic variable was then added to the demographic groups' test scores, which is mathematically equivalent to lowering that group's cutoff by an equal amount. ${ }^{5}$ Using empirical data, Carman et al. $(2018,2020)$ found that, out of the various identification schemes, this cutoff adjustment procedure was the second most effective for producing a gifted program with a racial group composition that reflects the district's overall student population. (The procedure failed to produce a gifted program that was as diverse as one produced via the use of building-level local

\footnotetext{
${ }^{5}$ Carman et al. $(2018,2020)$ called this adjustment an "opportunity to learn modifier." However, they never presented any evidence that these average differences among demographic groups were actually caused by differences in opportunity to learn among the groups. This is why we use the more neutral term "cutoff adjustment," which does not imply why the adjustments may be needed.
} 
norms.) However, cutoff adjustments may also run into legal difficulties in jurisdictions that ban the consideration of race in educational decisions.

Indirect methods of adjusting cutoffs for racial groups adjust are much less likely to have legal problems. But they are also much less likely to be effective methods of diversifying gifted programs. The use of local building-level norms is an indirect way to adjust cutoff scores, albeit covertly. In the simulation for this article, cutoffs were always higher for schools that had a higher percentage of students from the group with the higher average score, while cutoffs were always lower for schools that had proportionately more students from the lower scoring group. Indeed, there was a direct mathematical relationship between the two variables such that a school's cutoff for admission into the gifted program decreased monotonically as the percentage of students from the lower scoring group increased. This tendency is also found in real data. In Peters et al.'s (2019) study, local building-level norms had the greatest impact on diversity in districts where minority populations were a larger percentage than average. This explains why local building-level norms were so effective at producing a diverse gifted program in Carman et al.'s $(2018,2020)$ studies of a school district that had $65.3 \%$ Hispanic students and $22.9 \%$ African American students.

This illustrates an important characteristic of indirect methods of cutoff adjustments: they are only effective at producing racially diverse gifted programs insomuch as the variable used adjust cutoffs is correlated with student race. This is why a fully segregated district could produce a gifted program with demographics that reflected the district population; the building a student attended was perfectly correlated with their race, thereby producing a gifted program that exactly matched the district's overall demographics. Conversely, in a fully integrated district, student race and the building they attended were completely uncorrelated-resulting in a gifted 
program that was the least racially diverse when school membership was used as a factor in selection. Likewise, in Carman et al.'s $(2018,2020)$ studies, cutoff adjustments were not perfectly correlated with race and failed to produce gifted programs that were as representative of the general school district as building-level norms did.

Another indirect method of adjusting cutoffs is to offer underrepresented students multiple methods of identification. Typical methods would include asking teachers to nominate additional underrepresented children, setting a lower cutoff score on an initial screening instrument, or requiring an admissions committee to give a second (or third) consideration to underrepresented students. There is no research on the effectiveness or consequences of these methods of increasing underrepresented students' presence in gifted programs. However, there is no reason to think that these procedures would decrease diversity in a gifted program.

All of these short-term methods for increasing diversity in gifted programs are imperfect. Some (e.g., quotas, race norming) may create legal problems if implemented. Others (like adjusting cutoffs for different racial groups and lowering standards for all students) may have limited effectiveness. But one problem — unmentioned so far-is present for all of them: the need to defend these procedures in the court of professional and/or public opinion. Lowering the definition of "giftedness" leaves school personnel open to accusations of watering down the academic rigor of a gifted program. Adjusting cutoff scores for some racial groups — whether indirectly or directly_-invites allegations of lowered expectations for underrepresented students and/or reverse discrimination. Using a test with smaller average score differences may result in using a test that is poorly aligned with gifted services. ${ }^{6}$ There is no perfect short-term solution to the problem of disproportionate representation in gifted programs. Consequentially, school

\footnotetext{
${ }^{6}$ Although is important to note that some abstract tests of cognitive ability — such as nonverbal matrix tests — also suffer from a poor alignment with the curriculum in a gifted program.
} 
personnel may have to decide the degree of disproportionality they are willing to live with and which adjustment procedure(s) they can defend to their peers, the courts, or a skeptical public.

\section{Equity vs. Excellence}

This discussion strikes at the heart of the equity vs. excellence conflict in gifted education. The uncomfortable reality of the present moment is that maximizing equity-i.e., proportionate representation-often requires selecting typically underrepresented students who meet lower academic standards. When this occurs, the population of gifted students becomes more academically heterogeneous, and it becomes more difficult to meet the academic needs of all selected children. Whenever average group differences are greater than zero, gifted programs that are demographically equitable will likely produce a disproportionate number of students from groups with lower average academic achievement compared to gifted students who belong to groups with overall higher average achievement.

Likewise, maximizing excellence under current conditions will likely result in programs that do not reflect the demographics of the general student population. Tables 3-6 and S2-S21 show that as a program becomes more elite (by admitting a smaller percentage of the top students within each school), the programs become more demographically imbalanced—unless a district is perfectly segregated (or there is no achievement gap between groups, as shown in Table 2).

Whenever average differences exist in achievement across groups, the excellence vs. equity conundrum will be present. It seems likely that the best way to completely meet the conflicting demands of equity and excellence in gifted education is to close the achievement gap among demographic groups. Increasing the academic achievement of students who belong to groups that — on average — have lower achievement improves both equity (by creating a larger 
pool of diverse students who meet selection criteria for gifted programs) and excellence (by bringing more students to high levels of academic performance). For this reason, gifted education scholars and personnel should be active parties in the effort to reduce achievement gaps within the general student population. Expending energy on other efforts to improve the diversity of gifted programs will likely yield comparatively fewer benefits.

\section{Limitations to the Simulation}

It is important to recognize the limits to this simulation. First, some readers may object to a simulation based on a single test score being used in gifted identification process because best practice is to use multiple measures to identify gifted children (Peters et al., 2016; Pfeiffer, 2002). However, if the multiple scores are all measures of the same underlying construct, then combining the scores will function to create a univariate composite of the students' level of the construct (e.g., McBee et al., 2014). If these scores are summed or averaged, then the resulting composite will have high reliability and will likely have the similar statistical and psychometric properties as a single score used for selection.

Some theoretical perspectives define "giftedness" as being multidimensional in nature and that it cannot be summed up as one score (e.g., Renzulli, 1978). Others see "giftedness" as being the end product of a developmental process that results in high ability (Subotnik et al., 2011). These are legitimate theoretical perspectives. However, when deciding who deserves the label of "gifted," it is still necessary to collapse multiple variables into a dichotomous label of "gifted" or "non-gifted." This reality means that our simulation still has applicability to these perspectives of giftedness.

Another limitation of these calculations is that the schools were assumed to be the same size. Dissimilarity indexes are influenced by the relative size of a district's schools, and lopsided 
enrollments across schools can greatly influence a district's level of integration. If a larger school is integrated and smaller schools are not, or if larger schools tend to have a larger proportion of minority students, then this can artificially drive the dissimilarity index down. When local building-level norms are applied to such districts, the results in terms of the gifted program's composition may be more akin to what was seen in highly segregated districts in our study than in the integrated districts where all schools have the same enrollment.

Likewise, the calculations are based on districts that have a 50-50 split in two demographic groups that both have a normal distribution of score. A more lopsided demographic composition or a severely non-normal distribution of scores may produce gifted groups with demographics, achievement gaps within the gifted population, and gifted/non-gifted dissimilarity indexes that differ from what is seen in this simulation. (Relative risks values will remain the same, however.) With some reasonable assumptions, the results from this simulation can be extrapolated to districts with other demographic ratios. ${ }^{7}$

Likewise, the simulated conditions of two demographic groups are simplistic. Some readers may be interested in the consequences of having average differences in scores among three (or more) demographic groups. We only simulated two groups because three of the five outcome metrics (i.e., mean difference within the gifted group, relative risk, and gifted/non-

\footnotetext{
${ }^{7}$ To find the percentage of students in Group B $\left(\operatorname{gifted}_{B}\right)$ who are selected for the gifted program, the formula is $\operatorname{gifted}_{B}=\left(R R_{B}\right)\left(\right.$ gifted $\left._{A}\right)$, where $R R_{B}$ is the relative risk for group A to be selected for a gifted program (available from the tables in this simulation) and gifted ${ }_{A}$ is the absolute probability that a member of Group A will be selected for the gifted program (which must be either known beforehand or estimated). With these numbers, it is possible to find the demographics of the gifted group with the percentage of gifted students belonging to Group A being gifted ${ }_{A}$ $/\left(\operatorname{gifted}_{A}+\operatorname{gifted}_{B}\right)$ and the percentage of gifted students belonging to Group B being gifted $/\left(\operatorname{gifted}_{A}+\operatorname{gifted}_{B}\right)$. Calculating the other results of the simulation (i.e., average $z$-score for students selected for a gifted program, average group mean difference between demographic groups among gifted students, and gifted/non-gifted dissimilarity index) are more complex and depend — partially—on district dissimilarity index, number of schools, and enrollment of schools within a district. Still, the general pattern will hold that increasing district-level integration will result in an increasingly non-representative gifted program, smaller mean differences between groups within the gifted population, and a higher gifted/non-gifted dissimilarity index.
} 
gifted dissimilarity index) can only be calculated with two groups at a time. Having additional groups would require $k(k-1) / 2$ values, where $k$ is the number of groups. Readers who wish to extrapolate to additional groups can apply this simulation's results by considering groups one pair at a time. For example, if Group A in a district scores $d=.30$ higher than Group B, which in turn outscores Group C by $d=.60$, then the Table 2 provides the information for comparing Groups A and B, and Table 3 provides the information for comparing groups B and C. Groups A and C can be compared using Table 4 (where $d=.90$ ) because that difference is the sum of the other two pairwise differences. The supplemental tables provide results for other differences (in .10 increments), ranging from $d=0$ to 2.0 .

However, departures from the ideals that form the basis of this simulation will not change the overall pattern found of more integrated districts having less representative gifted programs because this pattern is a result of building-level norms increasing proportionality to the extent that building membership is correlated with demographic group membership. When building membership and demographic group membership are weakly correlated or uncorrelated (i.e., when districts are increasingly integrated) an inverse relationship between district integration and gifted program integration will be present. The point of this simulation has not been to make exact predictions about the composition any particular district's gifted program; instead, the purpose has been to show how district integration and achievement gaps limit the capacity of local building-level norms to produce a gifted group that reflects the general demographics of a district.

\section{Conclusion}

In recent years, gifted education scholars have reached a consensus that using local norms to identify gifted students is best practice - a position that we endorse. However, it has become 
clear that "Implementing local norms is not a panacea for addressing all systematic causes of underrepresentation in gifted education" (Peters et al., 2019, p. 15). The simulation in this article shows that the degree to which a district is racially integrated limits the effectiveness of using local building-level norms to produce gifted programs with demographics that mirror a district's student population.

This article also shows how fundamental the achievement gap is as a cause of over- and underrepresentation in gifted programs. Furthermore, the achievement gap in the overall student population has consequences that extend far beyond the demographic makeup of gifted programs. Average achievement differences have implications for curriculum planning within gifted programs, investigating discriminatory gifted identification practices, and the equity vs. excellence dilemma. These consequences show that the concerns of gifted education scholars and practitioners are aligned with the concerns of other educational professionals. Working with colleagues outside of gifted education - and providing them with insights and practices that the gifted education community has developed—can help both groups accomplish their common goals and serve the needs of all students. 


\section{References}

Calvin, A. (2000). Use of standardized tests in admissions in postsecondary institutions of higher education. Psychology, Public Policy, and Law, 6(1), 20-32. https://doi.org/10.1037/1076-8971.6.1.20

Carman, C. A., Walther, C. A. P., \& Bartsch, R. A. (2018). Using the Cognitive Abilities Test (CogAT) 7 nonverbal battery to identify the gifted/talented: An investigation of demographic effects and norming plans. Gifted Child Quarterly, 62(2), 193-209. https://doi.org/10.1177/0016986217752097

Carman, C. A., Walther, C. A. P., \& Bartsch, R. A. (2020). Differences in using the Cognitive Abilities Test (CogAT) 7 nonverbal battery versus the Naglieri Nonverbal Ability Test (NNAT) 2 to identify the gifted/talented. Gifted Child Quarterly, 64(3), 171-191. https://doi.org/10.1177/0016986220921164

Curran, F. C., \& Kellogg, A. T. (2016). Understanding science achievement gaps by race/ethnicity and gender in kindergarten and first grade. Educational Researcher, 45(5), 273-282. https://doi.org/10.3102/0013189x16656611

de Brey, C., Musu, L., McFarland, J., Wilkinson-Flicker, S., Diliberti, M., Zhang, A., Branstetter, C., \& Wang, X. (2019). Status and trends in the education of racial and ethnic groups 2018 (NCES Publication No. 2019-038). U.S. Department of Education, National Center for Educational Statistics. https://nces.ed.gov/pubs2019/2019038.pdf

Deary, I. J., Thorpe, G., Wilson, V., Starr, J. M., \& Whalley, L. J. (2003). Population sex differences in IQ at age 11: The Scottish mental survey 1932. Intelligence, 31(6), 533542. https://doi.org/10.1016/s0160-2896(03)00053-9 
Feingold, A. (1992). Sex differences in variability in intellectual abilities: A new look at an old controversy. Review of Educational Research, 62(1), 61-84. https://doi.org/10.3102/00346543062001061

Ford, D. Y. (2014). Segregation and the underrepresentation of Blacks and Hispanics in gifted education: Social inequality and deficit paradigms. Roeper Review, 36(3), 143-154. https://doi.org/10.1080/02783193.2014.919563

Frisby, C. L. (2013). Meeting the psychoeducational needs of minority students: Evidence-based guidelines for school psychologists and other school personnel. John Wiley \& Sons.

Fry, R. (2009). The rapid growth and changing complexion of suburban public schools. Pew Research Center.

Grissom, J. A., \& Redding, C. (2016). Discretion and disproportionality: Explaining the underrepresentation of high-achieving students of color in gifted programs. AERA Open, 2(1), Article 2332858415622175. https://doi.org/10.1177/2332858415622175

James, D. R., \& Taeuber, K. E. (1985). Measures of segregation. Sociological Methodology, 15, 1-32. https://doi.org/10.2307/270845

Jensen, A. R. (1980). Précis of bias in mental testing. Behavioral and Brain Sciences, 3(3), 325333. https://doi.org/10.1017/S0140525X00005161

Jensen, A. R. (1985). The nature of the black-white difference on various psychometric tests: Spearman's hypothesis. Behavioral and Brain Sciences, 8(2), 193-219. https://doi.org/10.1017/S0140525X00020392

Jensen, A. R. (1998). The g factor: The science of mental ability. Praeger. 
Kalogrides, D., \& Loeb, S. (2013). Different teachers, different peers: The magnitude of student sorting within schools. Educational Researcher, 42(6), 304-316. https://doi.org/10.3102/0013189x13495087

Knott, K. (2021, June 14). 86\% of Charlottesville students in grades 3-11 are identified as gifted. The Daily Progress. https://dailyprogress.com/news/local/education/86-of-charlottesvillestudents-in-grades-3-11-are-identified-as-gifted/article_49ff3982-cd5e-11eb-8776eb3c4344ae73.html

Lakin, J. M. (2018). Making the cut in gifted selection: Score combination rules and their impact on program diversity. Gifted Child Quarterly, 62(2), 210-219. https://doi.org/10.1177/0016986217752099

Lamb, K. N., Boedeker, P., \& Kettler, T. (2019). Inequities of enrollment in gifted education: A statewide application of the $20 \%$ equity allowance formula. Gifted Child Quarterly, 63(4), 205-224. https://doi.org/10.1177/0016986219830768

Lipsey, M. W., Puzio, K., Yun, C., Heberg, M. A., Steinka-Fry, K., Cole, M. W., Roberts, M., Anthony, K. S., \& Busick, M. D. (2012). Translating the statistical representation of the effects of education interventions into more readily interpretable forms (NCES Publication No. 2013-3000). U.S. Department of Education, National Center for Educational Statistics. https://ies.ed.gov/ncser/pubs/20133000/pdf/20133000.pdf

Little, M. (2017). Racial and socioeconomic gaps in executive function skills in early elementary school. Educational Researcher, 46(2), 103-109. https://doi.org/10.3102/0013189x17698700 
Makel, M. C., Kell, H. J., Lubinski, D., Putallaz, M., \& Benbow, C. P. (2016). When lightning strikes twice: Profoundly gifted, profoundly accomplished. Psychological Science, 27(7), 1004-1018. https://doi.org/10.1177/0956797616644735

McBee, M. T., Peters, S. J., \& Waterman, C. (2014). Combining scores in multiple-criteria assessment systems: The impact of combination rule. Gifted Child Quarterly, 58(1), 6989. https://doi.org/10.1177/0016986213513794

Murray, C. (2021). Facing reality: Two truths about race in America. Encounter Books.

Naglieri, J. A., \& Ford, D. Y. (2003). Addressing underrepresentation of gifted minority children using the Naglieri Nonverbal Ability Test (NNAT). Gifted Child Quarterly, 47, 155-160. https://doi.org/10.1177/001698620304700206

Olszewski-Kubilius, P., Steenbergen-Hu, S., Thomson, D., \& Rosen, R. (2017). Minority achievement gaps in STEM: Findings of a longitudinal study of Project Excite. Gifted Child Quarterly, 61(1), 20-39. https://doi.org/10.1177/0016986216673449

Peters, S. J., \& Engerrand, K. G. (2016). Equity and excellence: Proactive efforts in the identification of underrepresented students for gifted and talented services. Gifted Child Quarterly, 60(3), 159-171. https://doi.org/10.1177/0016986216643165

Peters, S. J., Matthews, M. S., McBee, M. T., \& McCoach, D. B. (2014). Beyond gifted education: Designing and implementing advanced academic programs. Prufrock Press.

Peters, S. J., Rambo-Hernandez, K., Makel, M. C., Matthews, M. S., \& Plucker, J. A. (2019). Effect of local norms on racial and ethnic representation in gifted education. AERA Open, 5(2), 1-18. https://doi.org/10.1177/2332858419848446 
Petersen, N. S., \& Novick, M. R. (1976). An evaluation of some models for culture-fair selection. Journal of Educational Measurement, 13(1), 3-29. https://doi.org/10.1111/j.1745-3984.1976.tb00178.x

Pfeiffer, S. I. (2002). Identifying gifted and talented students: Recurring issues and promising solutions. Journal of Applied School Psychology, 19(1), 31-50. https://doi.org/10.1300/J008v19n01_03

Rambo-Hernandez, K. E., \& Warne, R. T. (2015). Measuring the outliers: An introduction to out-of-level testing with high-achieving students. Teaching Exceptional Children, 47(4), 199-207. https://doi.org/10.1177/0040059915569359

Reardon, S. F., \& Portilla, X. A. (2016). Recent trends in income, racial, and ethnic school readiness gaps at kindergarten entry. AERA Open, 2(3), Article 2332858416657343. https://doi.org/10.1177/2332858416657343

Renzulli, J. S. (1978). What makes giftedness? Reexamining a definition. Phi Delta Kappan, 60, 180-184, 261. https://doi.org/10.1177/003172171109200821

Sander, R. H., \& Taylor, S., Jr. (2012). Mismatch: How affirmative action hurts students it's intended to help, and why universities won't admit it. Basic Books.

Subotnik, R. F., Olszewski-Kubilius, P., \& Worrell, F. C. (2011). Rethinking giftedness and gifted education: A proposed direction forward based on psychological science. Psychological Science in the Public Interest, 12(1), 3-54. https://doi.org/10.1177/1529100611418056

Swanson, J. D. (2006). Breaking through assumptions about low-income, minority gifted students. Gifted Child Quarterly, 50(1), 11-25. https://doi.org/10.1177/001698620605000103 
te Nijenhuis, J., van den Hoek, M., \& Armstrong, E. L. (2015). Spearman's hypothesis and Amerindians: A meta-analysis. Intelligence, 50, 87-92. https://doi.org/10.1016/j.intell.2015.02.006

Warne, R. T. (2016a). Five reasons to put the $g$ back into giftedness: An argument for applying the Cattell-Horn-Carroll theory of intelligence to gifted education research and practice. Gifted Child Quarterly, 60(1), 3-15. https://doi.org/10.1177/0016986215605360

Warne, R. T. (2016b). Testing Spearman's hypothesis with Advanced Placement examination data. Intelligence, 57, 87-95. https://doi.org/10.1016/j.intell.2016.05.002

Warne, R. T. (2020). In the know: Debunking 35 myths about human intelligence. Cambridge University Press.

Warne, R. T. (2021). Statistics for the social sciences: A general linear model approach (2nd ed.). Cambridge University Press.

Warne, R. T. (in press). Analyzing disproportionate representation in gifted education: Identification procedures, proximal causes, distal causes, and theoretical causes. Gifted Child Quarterly.

Warne, R. T., Anderson, B., \& Johnson, A. O. (2013). The impact of race and ethnicity on the identification process for giftedness in Utah. Journal for the Education of the Gifted, 36(4), 487-508. https://doi.org/10.1177/0162353213506065

Worrell, F. C., \& Dixson, D. D. (2018). Recruiting and retaining underrepresented gifted students. In S. I. Pfeiffer (Ed.), Handbook of giftedness in children: Psychoeducational theory, research, and best practices (pp. 209-226). Springer International Publishing. https://doi.org/10.1007/978-3-319-77004-8_13 
Worrell, F. C., Subotnik, R. F., Olszewski-Kubilius, P., \& Dixson, D. D. (2019). Gifted students. Annual Review of Psychology, 70(1), 551-576. https://doi.org/10.1146/annurev-psych010418-102846

Yoon, S. Y., \& Gentry, M. (2009). Racial and ethnic representation in gifted programs: Current status of and implications for gifted Asian American students. Gifted Child Quarterly, 53(2), 121-136. https://doi.org/10.1177/0016986208330564 